\title{
Hugoniot Equation of State of Polymers
}

\section{RECEIVED \\ AUG 281995 \\ OSTI}

\section{Los Alamos}

Los Alamos National Laboratory is operated by the University of California for the United States Department of Energy under contract W-7405-ENG-36. 
An Affirmative Action/Equal Opportunity Employer

This report was prepared as an account of work sponsored by an agency of the United States Government. Neither The Regents of the University of California, the United States Government nor anyagency thereof, nor any of their employees, makes any tuarranty, express or implied, or assumes any legal liability or responsibility for the accuracy, completeness, or usefulness of any information, apparatus, product, or process disclosed, or represents that its use would not infringe privately owned rights. Reference herein to any specific commercial product, process, or service by trade name, trademark, manufacturer, or otherwise, does not necessarily constitute or imply its endorsement recommendation, or favoring by The Regents of the University of Califormia, the United States Government, or any agency thereof. The views and opinions of authors expressed herein do not necessarily state or reflect those of The Regents of the University of California, the United States Government, or any agency thereof. 


\section{DISCLAIMER}

Portions of this document may be illegible in electronic image products. Images are produced from the best available original document. 


\section{Hugoniot Equation of State of Polymers}

William J. Carter

Stanley P. Marsh 



\section{PREFACE}

This report was put together in 1977 but for several reasons has not been made readily accessible. The data was published in the LASL Shock-Data book in 1980 edited by S. Marsh. However, the data regarding the Hugoniot cusps and Carter and Marsh's ideas concerning the reason for the cusps have not been available. We have taken it upon ourselves to make an LA-series report as it would have appeared in 1977 so the data and ideas expressed by the authors can appear in a coherent form. Although other shock work has been done on polymers since then, no definitive information regarding the real time chemistry is yet available, hence these authors' ideas deserve a modern consideration.-J. N. Fritz and S. Sheffield. 


\title{
HUGONIOT EQUATION OF STATE OF POLYMERS
}

\author{
by
}

\author{
William J. Carter and Stanley P. Marsh
}

\begin{abstract}
The Hugoniot equations of state of a large number of representative polymers have been obtained. Two aspects of the results are particularly striking:

a) The $u_{s}\left(u_{p}\right)$ Hugoniots of all the polymers extrapolate to bulk sound velocities higher than the ultrasonic values, an indication of a rapidly varying rate of change of compressibility in this region. This is attributed both to the two-dimensional nature of polymer compression and to the form of the interchain interaction potential.

b) A relatively high pressure transformation (in the range $20-30 \mathrm{GPa}$ ), characterized by a change in slope of the $u_{s}\left(u_{p}\right)$ Hugoniot and sometime by a large volume change as well, is observed for all of the polymers. This transformation is probably associated with pressure-induced cross bonding. In particular, for those polymers which contain rings in their monomer structure and which display the largest volume change at transformation, it is proposed that carbon-carbon covalent bonds along chains are broken and tetragonal bonds between chains are formed in a manner analogous to the graphite-diamond transformation.
\end{abstract}




\section{INTRODUCTION}

Although polymers are widely used in engineering applications involving high pressure, there exist remarkably little data on their compressive behavior above a few GPa. There are some static data available in the range $0-2$ GPa for a few materials ${ }^{1}$, and considerable shock-wave data have been amassed for materials such as polyethylene and polymethylmethacrylate ${ }^{2}$ and epoxy ${ }^{3}$. There have been phase changes reported in a few polymers in the low-pressure range, notably the spiral transformation in Teflon at about one half GPa, which have been thoroughly studied using $\mathrm{x}$-ray diffraction ${ }^{4}, 5$. However, the general lack of dynamic high-pressure data has prompted the present study. The polymeric solids studied (which include both thermosetting and thermoplastic types) are listed in Table I along with pertinent thermophysical properties at zero pressure. Most of these materials were fabricated by Group CMB-6 of Los Alamos under conditions which are both known and reproducible. An exception was the polyphenylquinoxaline, an experimental, highly aromatic polymer which was kindly supplied by the United States Naval Ordnance Laboratory ${ }^{6}$. A few other materials were obtained in bulk solid-form from commercial sources.

\section{EXPERIMENTAL PROCEDURE}

Standard shock-wave techniques ${ }^{3}$, which utilize high explosives to generate the shocks and streak cameras to record the wave-arrival times, were used to obtain shock-wave velocities, $u_{s}$, through the samples. The impedance-match technique then sufficed to determine the associated material velocities, $u_{p}$. The well-known Rankine-Hugoniot relationships between these experimentally determined kinematic quantities and the pressure $P$, volume $V$, and energy $E$ then determine the equation of state along the locus of final shock states, or the Hugoniot. Although energy is known along these curves, the temperature can only be crudely established because the volume dependence of the specific heat and the Grüneisen parameter for polymers is not well known 7 . Hence, quantitative thermodynamic calculations are of dubious value. In particular, no attempt was made to calculate off-Hugoniot loci, such as isotherms. Our compression curves include temperature effects and therefore will lie above those determined by static means, especially in the higher pressure regions.

Sound velocities at zero pressure were obtained using a pulse-echo technique which has also been previously described ${ }^{3}$. Both longitudinal $\left(c_{l}\right)$ and transverse $\left(c_{t}\right)$ modes were measured and combined to form the "bulk sound speed" $c_{b}$ through the relation $c_{b}^{2}=c_{l}^{2}-(4 / 3) c_{t}^{2}$. This may not be appropriate for materials which have a high enough crystallinity to be non-isotropic on a macroscopic scale. However, even for linear polyethylene, rotation of the sample in its holder resulted in no noticeable anisotropy. Shear-mode echoes were sometimes difficult to detect so that first-arrival times through samples of varying thickness were used when necessary. These velocities are listed in Table I. 


\section{RESULTS AND DISCUSSION}

The Hugoniot curves are reasonably well defined for each of the polymers considered here, and the results are summarized and listed in Table I. Here, the data have been fitted to segmented lines of the form $u_{s}=c_{0}+s u_{p}$ by the method of least squares, with the range of validity of each fit given by minimum and maximum values of $u_{p}$. Listed also are the transition pressures and compressions (discussed below) and an estimate of the associated volume change at transition. A detailed listing of the data points is given in the appendix. Figure 1 shows a typical Hugoniot of a

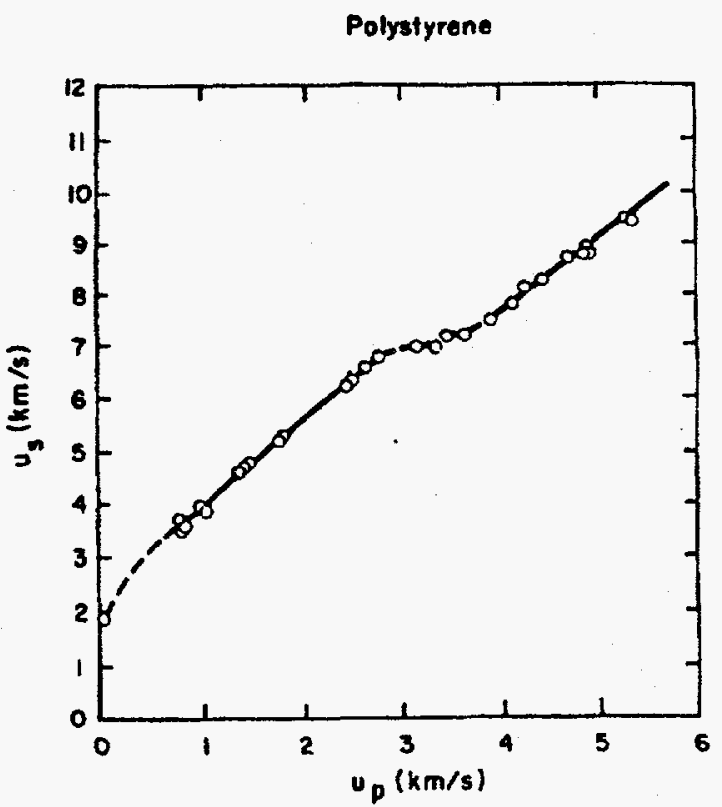

(a)

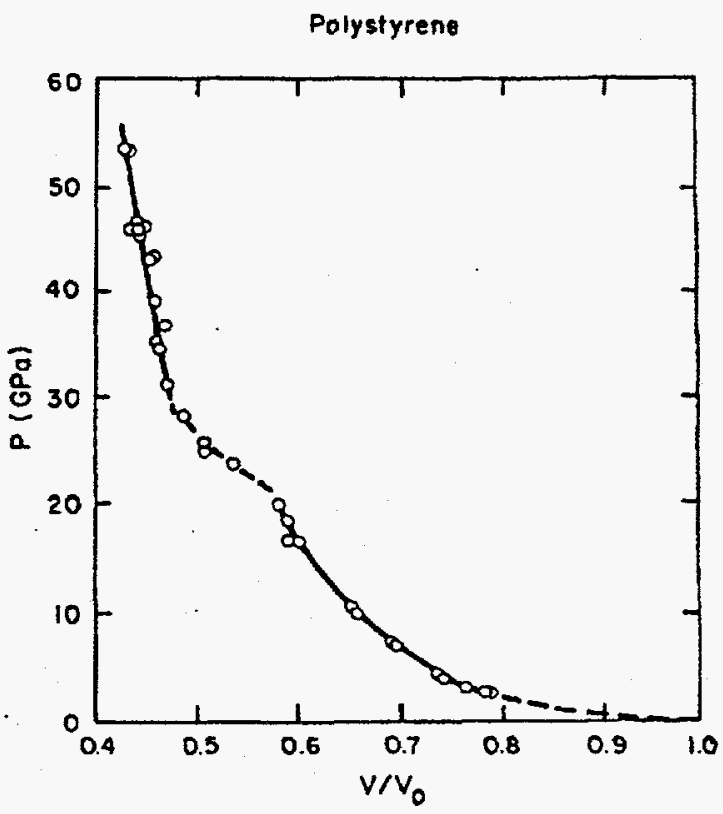

(b)

Figure 1: Hugoniot $u_{s}\left(u_{p}\right)(\mathrm{a})$ and $P\left(V / V_{0}\right)$ (b) data for polystyrene. The Hugoniot for this material is typical for most of the remaining polymers both in number of data points and quality of data as indicated by scatter from a linear fit. It also shows the two characteristics found for all polymers: a sound velocity that lies well below the Hugoniot intercept and a well-defined but pressure-dependent transformation which begins at $20 \mathrm{GPa}$.

polymer (polystyrene) in both the $u_{s}-u_{p}$ and $P-V$ planes, and illustrates two of the features which are prominent in the Hugoniots of most of the remaining materials. These features warrant further discussion and will be considered in detail.

The first such feature common to all the polymer Hugoniots is the failure of the high-pressure data to extrapolate to the zero-pressure ultrasonic measurements. This is also strikingly evident in a comparison between $c_{b}$, the ultrasonic sound speed, and the corresponding values of $c_{0 a}$, the zero-pressure intercept of the lower segment of the Hugoniot, from Table I. Ordinarily, this behavior is an indication either of a low-pressure phase change or of a high shear-strength which does not allow the one-dimensional Hugoniot to relax to the hydrostat in times short compared to the measurement interval. With a few exceptions, the static data do not show any phase 
Table I. Equation of State Properties of Polymers

\begin{tabular}{|c|c|c|c|c|c|c|c|c|c|c|c|c|c|c|c|}
\hline \multirow[t]{2}{*}{ Material } & \multicolumn{4}{|c|}{ Properties at Zero Pressure } & \multicolumn{6}{|c|}{ Summary of Hugoniot Results } & \multicolumn{5}{|c|}{ Transformation Parameters } \\
\hline & $\begin{array}{c}\rho_{o} \\
\left(\mathrm{~g} / \mathrm{cm}^{3}\right)\end{array}$ & $\begin{array}{c}c_{\ell} \\
(\mathrm{km} / \mathrm{s})\end{array}$ & $\begin{array}{c}c_{t} \\
(\mathrm{~km} / \mathrm{s})\end{array}$ & $\begin{array}{c}c_{b} \\
(\mathrm{~km} / \mathrm{s})\end{array}$ & $\begin{array}{c}c_{0 a} \\
(\mathrm{~km} / \mathrm{s})\end{array}$ & $s_{a}$ & $\begin{array}{r}u_{\text {prange }} \\
(\mathrm{km} / \mathrm{s})\end{array}$ & $\begin{array}{c}c_{0 b} \\
(\mathrm{~km} / \mathrm{s})\end{array}$ & $s_{b}$ & $\begin{array}{c}u_{\text {prange }} \\
(\mathrm{km} / \mathrm{s})\end{array}$ & $\begin{array}{c}P_{t} \\
(\mathrm{GPa})\end{array}$ & $\left(V / V_{0}\right)_{t}$ & $\begin{array}{c}V_{t} \\
\left(\mathrm{~cm}^{3} / \mathrm{g}\right)\end{array}$ & $\underset{(\%)}{(\Delta V / V)_{t}}$ & $\begin{array}{c}(P \Delta V)_{t} \\
(\mathrm{~J} / \mathrm{mg})\end{array}$ \\
\hline \multicolumn{16}{|l|}{ Polyethylene } \\
\hline linear & 0.954 & 2.462 & 1.014 & 2.166 & 2.86 & 1.57 & $0.7-3.2$ & 3.27 & 1.43 & $3.4-5.3$ & 24.7 & .592 & .0027 & 0.4 & 0.066 \\
\hline branched & 0.916 & 2.043 & 0.659 & 1.896 & 2.61 & 1.63 & $0.7-2.9$ & 3.17 & 1.41 & $3.0-4.8$ & 19.5 & .605 & .0054 & 0.8 & 0.106 \\
\hline olyvinyl chloride & 1.376 & 2.296 & 1.080 & 1.928 & 2.33 & 1.50 & $0.7-2.6$ & 2.34 & 1.46 & $2.7-4.9$ & 22.3 & .583 & .0061 & 1.4 & 0.136 \\
\hline Polyvinylidene fluoride & 1.767 & 2.097 & 0.850 & 1.853 & 2.58 & 1.58 & $0.6-2.6$ & 2.98 & 1.39 & $2.8-4.6$ & 31.7 & .609 & .0042 & 1.2 & 0.134 \\
\hline Polychlorotrifluorethylene & 2.133 & 1.744 & 0.769 & 1.501 & 2.05 & 1.66 & $0.6-2.9$ & 2.51 & 1.51 & $3.0-4.1$ & 43.7 & .575 & & & - \\
\hline Polytetrafluoroethylene & 2.151 & 1.230 & 0.406 & 1.139 & 1.68 & 1.79 & $0.6-2.8$ & 2.08 & 1.62 & $2.9-3.7$ & 41.6 & .580 & .0029 & 1.1 & 0.123 \\
\hline olyp & 0.904 & 2.583 & 1.256 & 2.137 & 2.86 & 1.49 & $0.7-3.2$ & 3.22 & 1.38 & $3.2-$ & 21 & .583 & - & - & \\
\hline Poly-(4-methyl-1-pentene) & 0.830 & 2.193 & 1.077 & 1.806 & 2.07 & 1.59 & $0.5-3.0$ & 2.42 & 1.46 & $3.1-5.5$ & 17.2 & .561 & .0041 & 0.6 & 0.070 \\
\hline Polyamide & 1.140 & 2.535 & 1.078 & 2.208 & 2.67 & 1.69 & $0.8-2.6$ & 3.89 & 1.18 & $2.8-5.2$ & 20.8 & .633 & .0060 & 1.1 & 0.126 \\
\hline Polym & 1.186 & 2.688 & 1.304 & 2.227 & 2.59 & 1.52 & $0.3-2.9$ & 2.90 & 1.33 & $3.4-5.3$ & 26.2 & .578 & .0166 & 3.4 & 0.433 \\
\hline Polystyrene & 1.046 & 2.306 & 1.144 & 1.890 & 2.34 & 1.58 & $0.7-2.8$ & 1.92 & 1.43 & $3.9-5.4$ & 19.8 & .587 & .0684 & 12.2 & 1.356 \\
\hline ellu & 1.255 & 2.450 & 1.153 & 2.057 & 2.29 & 1.55 & $0.6-2.6$ & 2.27 & 1.41 & & 20 & .589 & .0274 & 1.9 & .568 \\
\hline Epoxy & 1.192 & 2.641 & 1.177 & 2.264 & 2.69 & 1.51 & $0.4-2.8$ & 2.88 & 1.35 & 3.6 & 23.1 & .597 & .0197 & 3.9 & 0.454 \\
\hline Phenolic & 1.385 & 3.058 & 1.594 & 2.442 & 2.98 & 1.39 & $0.6-2.6$ & 2.05 & 1.55 & $3.0-5.0$ & 23.2 & .609 & .0296 & 6.7 & 0.687 \\
\hline Phenox & 1.178 & 2.506 & 1.069 & 2.173 & 2.63 & 1.54 & $0.6-2.6$ & 2.46 & 1.40 & $3.6-5.2$ & 21.7 & .602 & .0373 & 7.3 & 0.808 \\
\hline Polycarbonate & 1.196 & 2.187 & 0.886 & 1.933 & 2.33 & 1.57 & $0.4-2.6$ & 2.06 & 1.39 & $3.6-5.2$ & 20.0 & .593 & .0566 & 11.4 & 1.131 \\
\hline Polyester & 1.217 & 2.528 & 1.262 & 2.066 & 2.57 & 1.49 & $0.7-2.6$ & 2.16 & 1.41 & 3.4 & 20.3 & .596 & .0434 & 8.8 & 0.879 \\
\hline Polyimide & 1.414 & 2.723 & 1.217 & 2.332 & 2.66 & 1.48 & $0.6-2.2$ & 0.93 & 1.64 & $3.4-4.6$ & 17.8 & .633 & .0908 & 20.3 & 1.615 \\
\hline \multicolumn{16}{|l|}{ Polyphenylquinoxyline (NOL) } \\
\hline non-cro & 1.206 & 2.703 & 1.269 & 2.271 & 2.46 & 1.48 & $0.7-2.2$ & 1.72 & 1.44 & 3.5 & 15.7 & .612 & .0672 & 13.3 & 1.058 \\
\hline 35 & 1.206 & 2.457 & 1.158 & 2.061 & 2.46 & 1.48 & $0.7-2.5$ & 1.72 & 1.44 & $3.5-5.3$ & 18.0 & .598 & .0657 & 13.3 & 1.183 \\
\hline & 1.235 & 2.249 & 0.930 & 1.976 & 2.35 & 1.55 & $0.7-2.4$ & 1.58 & 1.51 & $3.4-5.1$ & 18.5 & .603 & .0628 & 12.9 & 1.160 \\
\hline Polyurethane & 1.265 & 2.390 & 1.030 & 2.068 & 2.54 & 1.57 & $0.6-2.6$ & 2.25 & 1.47 & $3.6-5.1$ & 21.7 & .607 & .0353 & 7.3 & 0.768 \\
\hline
\end{tabular}


changes in this low-pressure region. It is known that some polymers become quite brittle upon impact, but capacitor and magnetic probe free-surface records show that the Hugoniot elastic limit of polymers is immeasurably small. Early dynamic experiments by Evans and Schmidt ${ }^{8}$ on polymethylmethacrylate also showed considerable curvature at the low-pressure end of the $u_{s}-u_{p}$ locus and it was surmised that this effect would appear in other polymers as well. Considerable theoretical progress has been made in understanding this behavior, at least for the simple case of polyethylene, by Pastine ${ }^{9,10}$. His development depends on the fact that the forces between adjacent chains in a polymer are at least an order of magnitude smaller than the forces along the backbone ${ }^{11}$. Hence, the initial compression, assuming hydrostaticity, will be of a two-dimensional rather than a three-dimensional nature on a microscope scale, with the distance between chains decreasing but length along the backbone remaining relatively unaffected. That is, to obtain the initial compressibility of a polymer it suffices to specify only the interchain forces (and, of course, the geometrical arrangements). For polyethylene, and probably for most polymers as well, the dominant repulsive forces are those between non-bonded nearest-neighbor hydrogens and the dominant attractive forces are of the London dispersion (van der Waals) types. That is, the potential is of the general form 12

$$
\phi=A e^{-r / r_{0}}-B / r^{6}
$$

where $r$ is the nearest-neighbor hydrogen distance and the individual potentials must be summed in a manner appropriate for the crystal structure. It is assumed by Pastine that this relation holds for amorphous polyethylene as well, except that the exponential term takes a slightly different form. Straightforward calculation of the pressure from this potential, assuming that the polymer is compressible in two dimensions only, then yields a $P(V)$ relation which shows considerable curvature when transformed to the $u_{s}-u_{p}$ plane. Similar low-pressure behavior can be expected for all polymers provided only that the dominant forces determining initial compression are those between non-bonded hydrogens in neighboring chains.

Experimental quantitative verification of these ideas is found in the Hugoniot data for polyethylene. We have examined two densities of polyethylene representing degrees of crystallinity of approximately $90 \%$ and $55 \%$. The low-pressure Hugoniot data are shown in Fig. 2, along with the theoretical Hugoniots derived for these densities from the purely crystalline and purely amorphous calculations of Pastine. The agreement is seen to be excellent. Also shown are the static data of Warfield $^{1}$ for the highly crystalline material, corrected from an isothermal to an isentropic intercept but with no shock heating included, and Bridgeman's uncorrected data ${ }^{13}$ on normal density polyethylene. These data lie in precisely the region of curvature of most interest, and again the agreement with theory is quite good. It is tempting to extend these ideas, at least qualitatively, to account for the curvature in the lower part of the $u_{s}\left(u_{p}\right)$ Hugoniots of other polymers. This is probably justified in general. However, it should be remembered that low-pressure phase changes do occur in a few polymers. There also are alternative theories which would yield equivalent results; for example, the elastic-plastic flow model proposed by Schmidt and Evans ${ }^{8}$, in which yielding oc- 


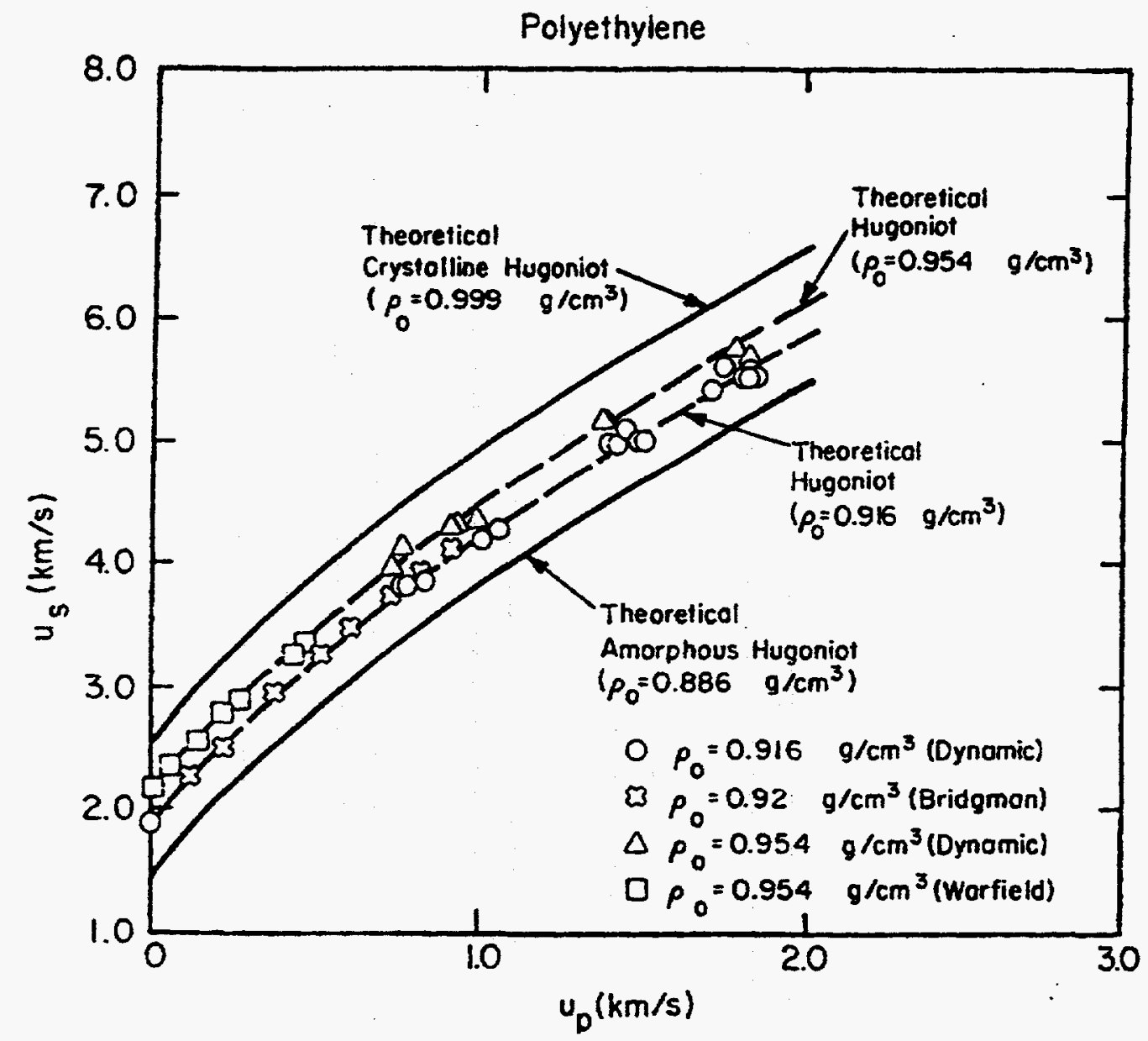

Figure 2: Low-pressure data for polyethylene. The lines (solid and dashed) are the theoretical calculations based on the work of Pastine, while the symbols are either dynamic data or static data transformed to the $u_{s}-u_{p}$ plane through the conservation relations. The agreement between theory and the various experimental data is excellent.

curs in a complex manner over a range of stresses and cannot be assigned a definite value, also predicts extreme curvature at low particle velocities. These possibilities should be further explored, but will probably require use of more sophisticated experimental shock-wave diagnostic techniques than have been used here.

A high-pressure $(20-30 \mathrm{GPa})$, high-temperature $(2000 \mathrm{~K})$ phase transformation is the second feature common to all the polymers with the possible exception of two of the fluoroplastics. For many materials the associated volume change is extremely large. Clearly, this is not a polymorphic transformation in the usual crystallographic sense, in that the transformation parameters appear to be insensitive to the degree of crystallinity (polystyrene, for example, is completely amorphous). Since similar behavior is observed on both thermosetting plastics and thermoplastics, the possibility of the transformation being associated with either melting or vaporization appears to be ruled out. Furthermore, it is unlikely that the glass-transition phase line extends to this region of pressure 
and temperature, and our calculations support this expectation. Also, at least at low pressures, the glass transition is not normally accompanied by a measurable volume change and there is little reason to expect this situation to change at higher pressures.

The most likely explanation of the nature of this transformation lies in an extension of the ideas already encountered at low pressures. At zero pressure, the elastic constants of polyethylene are known ${ }^{10}$ and $C_{33}$ (which defines compression along the backbone chain) is found to be at least a factor of 20 greater than the elastic constants associated with the other two crystal axes. However, this situation obviously cannot continue long before forces between chains other than the $\mathrm{H}-\mathrm{H}$ repulsion and van der Waals attraction come into play to stiffen the elastic constants $C_{11}$ and $C_{22}$, eventually making them comparable in magnitude to $C_{33}$. This implies that at sufficiently high pressures, the compressive behavior of polymers is similar to other normal three-dimensional solids, a fact which is reflected by the linear portion of the Hugoniot below the transition. At the transformation a major reordering on the molecular level is suggested since the volume changes for some polymers can evidently be quite large for this process. A possible type of such reordering is the following: when the distance between chains has been reduced by pressure to the point that interchain interactions become possible. This effect would be most pronounced in those polymers containing covalent-bonded carbon atoms in open structures such as benzene rings, since the breaking of covalent bonds within chains and subsequent reformation of tetravalent bonds between chains could lead to very large volume changes. Of course the concept of a chain-like structure in polymers loses meaning after this point, and one is dealing with a more normal solid above the transition consisting of an unspecified but definite array of the original constituent atoms.

These ideas are strengthened by the results shown in Table I. Here, an attempt has been made to estimate the volume contraction at transition for each of the polymers by measuring $\Delta V_{t}$ on the Hugoniot in the $P-V$ plane and ignoring such important thermodynamic details as the slope of the phase line and temperature effects. The enthalpy change $\Delta H_{t}$ at transition is similarly estimated as $(P \Delta V)_{t}$. Representative monomer structures for each of our polymers are shown schematically in Table II, and it is clear that there is a strong correlation between $\Delta H_{t}$ per unit volume from Table I and the number of benzene rings which are available for decomposition from Table II. This is further indicated in Fig. 3, where the $u_{s}\left(u_{p}\right)$ Hugoniots for five selected polymers are shown along with their representative monomer structures. Clearly, the transition becomes increasingly pronounced as the number of benzene rings in the backbone chain increases. The linear hydrocarbons and fluorocarbons, represented in Fig. 3 by polyethylene, display little more than a change in slope in the transition. Extremely open structures such as polysulfone and polyimide have transitions characterized by large volume changes. Polymers such as polystyrene also exhibit large volume changes, but the single ring in a styrene monomer is contained in a pendant chain and is therefore physically closer to the neighboring backbone fibers. In every case, the mixed-phase region of the $u_{s}\left(u_{p}\right)$ Hugoniot also displays a slightly positive slope which is probably indicative of a pressureenergy dependent transformation such as would be expected if our model is correct. 
Table II: Representative Idealized Monomer Structures.

$$
\begin{aligned}
& \text { polyethylene } \\
& \text { polyvinyl chloride }
\end{aligned}
$$

$$
-\mathrm{CH}_{2}-\underset{\mathrm{Cl}}{\mathrm{CH}}-
$$

polyvinylidene fluoride

$$
-\mathrm{CH}_{2}-\mathrm{CF}_{2}-
$$

polychlorotrifluoroethylene

$$
-\mathrm{CF}_{2}-\underset{\mathrm{Cl}}{\mathrm{CF}-}
$$

polytetraftuoroethylene

$$
-\mathrm{CF}_{2}-\mathrm{CF}_{2}-
$$

polyproprylene<smiles>CCC(C)C</smiles>

4-methyl-1-pentene-4

$$
\mathrm{CH}_{2}^{\mathrm{CH}}-\mathrm{CH}-\mathrm{CH}_{3}
$$$$
-\mathrm{CH}_{2}-\mathrm{CH}-
$$<smiles>CNCCNC(=O)CC(C)=O</smiles>

polyamide<smiles>CCC(C)(C)C(C)[O+]=O</smiles>

polystyrene<smiles>CCC(C)c1ccccc1</smiles>

polyimide

polyphenylquinoxyline

cellulose acetate<smiles>COC1OC(CO)C(C)C(O)C1O</smiles>

epoxy<smiles>COc1ccc(C2OC2C)cc1</smiles>

phenolic<smiles>Cc1ccc(Cc2cc(O)c(C)c(CO)c2)cc1O</smiles>

phenoxy

polycarbonate<smiles>CCC(O)COc1ccc(C(C)(C)c2ccc(OC)cc2)cc1</smiles><smiles>COC(=O)Oc1ccc(C(C)(C)c2ccc(C)cc2)cc1</smiles>

polyester

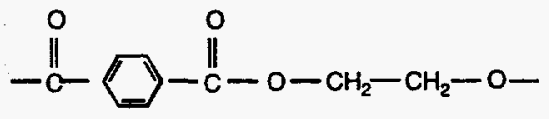<smiles>CN1C(=O)C2C(=O)C3C(C2=O)C12C(=O)N(c1ccc(Oc4ccccc4)cc1)C(=O)C32</smiles><smiles></smiles>

polysulfone<smiles>Cc1ccc(C(C)(C)c2ccc(Oc3ccc(S(=O)(=O)c4ccc(O[O-])cc4)cc3)cc2)cc1</smiles>

polyurethane<smiles>COC(=O)Nc1ccc(C)c(C)c1</smiles> 


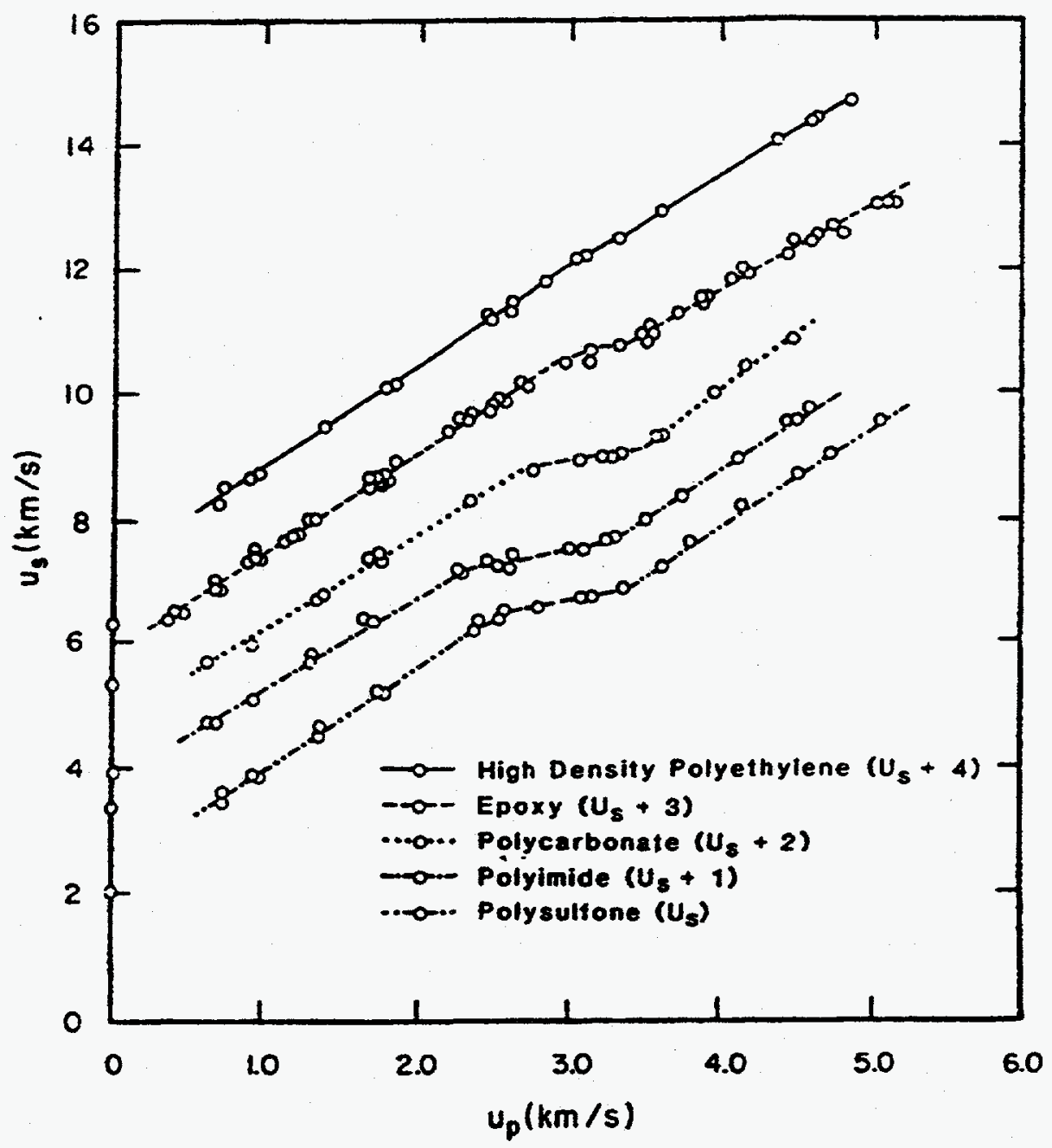

Figure 3: Hugoniot $u_{s}\left(u_{p}\right)$ data for several polymers. It is seen from Table II that, in general, those materials having more open units such as benzene rings in their monomer structure also display a more pronounced transition.

The picture which emerges then, is one in which the compression of a polymer under pressure is two-dimensional in nature until the interaction between chains becomes appreciable, after which the material compresses in a manner more typical of a three-dimensional solid. C-C interactions, primarily those between carbons in benzene rings belonging to different chains, gradually become important, eventually overriding the relatively weak covalent bonding of the ring structure, forming strong tetragonal bonds between chains, and effectively destroying the chain-like nature of the polymer in the process. This results in a volume change directly related to the number of such bonds broken and reformed. The process is one which is analogous to the well-known carbondiamond transformation, which also arises from the breaking of covalent bonds in a ring (lying in a plane rather than along a chain) and the formation of tetragonal bonds (between planes rather than between chains). This carbon-diamond transformation is also accompanied by an extremely 
large volume change.

It is very difficult to do quantitative first-principle calculations of the relative energies of these two phases. Although the C-C and C-H bonding energies are well-known at small compressions, these quantities are not known at compressions as large as 0.4 . Also, the density of rings subject to decomposition is required, a quantity which is not available for all our polymers. Finally, of course, many of these polymers contain open structures other than benzene rings which are also subject to decomposition and reformation, and the crystallographic arrangements of the final states are entirely unknown. We have attempted to verify or disprove our proposed transformation mechanism by recovering and checking the degree of cross bonding of samples shocked above the transformation pressure in expectation that the high-pressure phase may be metastable, but the recovery process has proved difficult and, so far, unsuccessful. If our model is correct, the high-pressure phase should have unique and possibly quite useful properties.

\section{REFERENCES}

1. R. W. Warfield, Poly. Eng. and Science, April (1966).

2. D. E. Munson and R. P. May, J. Appl. Phys. 43, 962 (1972).

3. R. G. McQueen, S. P. Marsh, J. W. Taylor, J. N. Fritz, and W. J. Carter, The equation of state of solids from shock wave studies, in High-Velocity Impact Phenomena, edited by R. Kinslow, chapter 7, Academic Press, New York, New York, 1970.

4. H. D. Flack, J. Poly. Science 10, 1799 (1972).

5. S. Hirakawa and T. Takemura, Jap. J. Appl. Phys. 8, 635 (1969).

6. W. Wrasidlo and J. M. Augl, J. Poly. Science A-1 7, 3393 (1969). (For a description of this material see Model VII.)

7. B. Wunderlich, J. Chem. Phys. 37, 1207 (1962).

8. D. N. Schmidt and M. W. Evans, Nature 206, 1248 (1965).

9. D. J. Pastine, J. Chem. Phys. 49, 3012 (1968).

10. D. J. Pastine, in Les Proprietes des Solides Sous Pression, edited by D. Block, 1970.

11. A. Muller, Proc. Roy. Soc. (London) A178, 227 (1941).

12. J. D. Boer, Physica 9, 363 (1942).

13. P. W. Bridgeman, Proc. Am. Acad. Arts Sci. 76, 71 (1948). 
APPENDIX. Hugoniot Data for Polymers

Shock velocities in the unknown $\left(u_{s}\right)$ and in the standard $\left(u_{s s t d}\right)$ are the measured quantities from which particle velocities $\left(u_{p}\right)$ are deduced from the continuity conditions. The remaining quantities pressure $(P)$, relative volume $\left(V / V_{o}\right)$, and density $(\rho)$ are obtained from the conservation relations. The integer value in the data tables under std indicates the standard used in the experiment. A 40 indicates a bulk value obtained from ultrasonic data. Data used for the standards in the experimental analysis are given in the following table. Off-Hugoniot EOS information required for calculating cross-curves for the standards is obtained by using the assumption that $\partial E / \partial P)_{V}=1 /(\rho \gamma)$ is a constant.

EOS Data for Standards

\begin{tabular}{lrrrrr}
\hline \hline Standard & std & $\begin{array}{c}\rho_{0} \\
\mathrm{~g} / \mathrm{cm}^{3}\end{array}$ & $\begin{array}{c}c_{0} \\
(\mathrm{~km} / \mathrm{s})\end{array}$ & $s$ & $\gamma_{0}$ \\
\hline 2024 Aluminum & 1 & 2.785 & 5.328 & 1.338 & 2.00 \\
921-T Aluminum & 2 & 2.833 & 5.041 & 1.420 & 2.10 \\
Copper & 10 & 8.930 & 3.940 & 1.489 & 1.99 \\
\hline
\end{tabular}

Polyethylene linear (Marlex EMN 6065)

\begin{tabular}{rrrrrrrr}
\hline $\begin{array}{c}\rho_{o} \\
\left(\mathrm{~g} / \mathrm{cm}^{3}\right)\end{array}$ & $\begin{array}{c}u_{s} \\
(\mathrm{~km} / \mathrm{s})\end{array}$ & $\begin{array}{c}u_{p} \\
(\mathrm{~km} / \mathrm{s})\end{array}$ & $\begin{array}{c}P \\
(\mathrm{GPa})\end{array}$ & $V / V_{o}$ & $\begin{array}{c}\rho \\
\left(\mathrm{g} / \mathrm{cm}^{3}\right)\end{array}$ & std & $\begin{array}{c}u_{s s t d} \\
(\mathrm{~km} / \mathrm{s})\end{array}$ \\
\hline 0.954 & 2.170 & 0.000 & 0.0 & 1.000 & 0.954 & 40 & 0.00 \\
0.954 & 3.985 & 0.721 & 2.7 & 0.819 & 1.165 & 1 & 5.93 \\
0.954 & 4.174 & 0.752 & 3.0 & 0.820 & 1.164 & 1 & 5.96 \\
0.954 & 4.355 & 0.921 & 3.8 & 0.788 & 1.210 & 1 & 6.11 \\
0.954 & 4.399 & 0.986 & 4.1 & 0.776 & 1.230 & 1 & 6.16 \\
0.954 & 5.147 & 1.398 & 6.9 & 0.728 & 1.310 & 1 & 6.54 \\
0.954 & 5.755 & 1.808 & 9.9 & 0.686 & 1.391 & 1 & 6.92 \\
0.954 & 5.779 & 1.824 & 10.1 & 0.684 & 1.394 & 1 & 6.94 \\
0.954 & 6.873 & 2.493 & 16.3 & 0.637 & 1.497 & 1 & 7.59 \\
0.954 & 6.835 & 2.497 & 16.3 & 0.635 & 1.503 & 1 & 7.59 \\
0.954 & 7.056 & 2.643 & 17.8 & 0.625 & 1.525 & 1 & 7.73 \\
0.954 & 7.076 & 2.657 & 17.9 & 0.625 & 1.527 & 1 & 7.75 \\
0.954 & 7.434 & 2.882 & 20.4 & 0.612 & 1.558 & 1 & 7.97 \\
0.954 & 7.760 & 3.098 & 22.9 & 0.601 & 1.588 & 1 & 8.19 \\
0.954 & 7.774 & 3.162 & 23.5 & 0.593 & 1.608 & 1 & 8.24 \\
0.954 & 8.079 & 3.377 & 26.0 & 0.582 & 1.639 & 1 & 8.46 \\
0.954 & 8.464 & 3.650 & 29.5 & 0.569 & 1.677 & 1 & 8.73 \\
0.954 & $\mathbf{9 . 0 7 2}$ & 4.136 & 35.8 & 0.544 & 1.754 & 1 & 9.21 \\
0.954 & $\mathbf{9 . 6 3 1}$ & 4.411 & 40.5 & 0.542 & 1.760 & 1 & 9.51 \\
0.954 & 9.946 & 4.697 & 44.6 & 0.528 & 1.808 & 1 & 9.79 \\
0.954 & $\mathbf{1 0 . 2 6 5}$ & 4.921 & 48.2 & 0.521 & 1.832 & 1 & 10.02 \\
0.954 & $\mathbf{1 0 . 7 3 6}$ & 5.305 & 54.3 & 0.506 & 1.886 & 1 & 10.40 \\
0.954 & 10.797 & 5.326 & 54.9 & 0.507 & 1.883 & 1 & 10.43 \\
\hline \hline
\end{tabular}

Polyethylene linear (Marlex 50)

\begin{tabular}{rrrrrrrr}
\hline \hline $\begin{array}{c}\rho_{o} \\
\left(\mathrm{~g} / \mathrm{cm}^{3}\right)\end{array}$ & $\begin{array}{c}u_{s} \\
(\mathrm{~km} / \mathrm{s})\end{array}$ & $\begin{array}{c}u_{p} \\
(\mathrm{~km} / \mathrm{s})\end{array}$ & $\begin{array}{c}P \\
(\mathrm{GPa})\end{array}$ & $V / V_{o}$ & $\begin{array}{c}\rho \\
\left(\mathrm{g} / \mathrm{cm}^{3}\right)\end{array}$ & std & $\begin{array}{r}u_{\text {sstd }} \\
(\mathrm{km} / \mathrm{s})\end{array}$ \\
\hline 0.954 & 2.130 & 0.000 & 0.0 & 1.000 & 0.954 & 40 & 0.00 \\
0.954 & 3.968 & 0.721 & 2.7 & 0.818 & 1.166 & 1 & 5.93 \\
0.954 & 4.131 & 0.754 & 3.0 & 0.818 & 1.167 & 1 & 5.96 \\
0.954 & 4.332 & 0.922 & 3.8 & 0.787 & 1.212 & 1 & 6.11 \\
0.955 & 4.361 & 0.987 & 4.1 & 0.774 & 1.235 & 1 & 6.16 \\
0.954 & 5.157 & 1.398 & 6.9 & 0.729 & 1.309 & 1 & 6.54 \\
0.954 & 5.764 & 1.807 & 9.9 & 0.686 & 1.390 & 1 & 6.92 \\
0.954 & 5.734 & 1.827 & 10.0 & 0.681 & 1.400 & 1 & 6.94 \\
0.954 & 6.862 & 2.494 & 16.3 & 0.637 & 1.499 & 1 & 7.59 \\
0.954 & 6.753 & 2.504 & 16.1 & 0.629 & 1.516 & 1 & 7.59 \\
0.954 & 6.939 & 2.654 & 17.6 & 0.618 & 1.545 & 1 & 7.73 \\
0.954 & 7.061 & 2.658 & 17.9 & 0.624 & 1.530 & 1 & 7.75 \\
0.954 & 7.371 & 2.888 & 20.3 & 0.608 & 1.569 & 1 & 7.97 \\
0.954 & 7.751 & 3.099 & 22.9 & 0.600 & 1.590 & 1 & 8.19 \\
0.954 & 7.788 & 3.161 & 23.5 & 0.594 & 1.606 & 1 & 8.24 \\
0.954 & 8.056 & 3.379 & 26.0 & 0.581 & 1.643 & 1 & 8.46 \\
0.954 & 8.493 & 3.647 & 29.6 & 0.571 & 1.672 & 1 & 8.73 \\
0.954 & 9.657 & 4.408 & 40.6 & 0.544 & 1.755 & 1 & 9.51 \\
0.954 & 9.978 & 4.675 & 44.5 & 0.531 & 1.795 & 1 & 9.77 \\
0.954 & 9.980 & 4.693 & 44.7 & 0.530 & 1.801 & 1 & 9.79 \\
0.954 & 10.390 & 4.906 & 48.6 & 0.528 & 1.807 & 1 & 10.02 \\
0.954 & 10.786 & 5.327 & 54.8 & 0.506 & 1.885 & 1 & 10.43 \\
\hline \hline
\end{tabular}


Polyethylene (branched Phillips)

\begin{tabular}{|c|c|c|c|c|c|c|c|}
\hline $\begin{array}{c}\rho_{o} \\
\left(\mathrm{~g} / \mathrm{cm}^{3}\right)\end{array}$ & $\begin{array}{c}u_{s} \\
(\mathrm{~km} / \mathrm{s})\end{array}$ & $\begin{array}{c}u_{p} \\
(\mathrm{~km} / \mathrm{s})\end{array}$ & $\begin{array}{c}P \\
(\mathrm{GPa})\end{array}$ & $V / V_{o}$ & $\begin{array}{c}\rho \\
\left(\mathrm{g} / \mathrm{cm}^{3}\right)\end{array}$ & std & $\begin{array}{c}u_{\text {sstd }} \\
(\mathrm{km} / \mathrm{s})\end{array}$ \\
\hline 0.916 & 1.896 & 0.000 & 0.0 & 1.000 & 0.916 & 40 & 0.00 \\
\hline 0.916 & 3.817 & 0.759 & 2.7 & 0.801 & 1.143 & 1 & 5.95 \\
\hline 0.918 & 4.001 & 0.764 & 2.8 & 0.809 & 1.135 & 1 & 5.96 \\
\hline 0.916 & 3.813 & 0.766 & 2.7 & 0.799 & 1.146 & 1 & 5.96 \\
\hline 0.918 & 3.853 & 0.831 & 2.9 & 0.784 & 1.170 & 1 & 6.01 \\
\hline 0.918 & 4.229 & 1.001 & 3.9 & 0.763 & 1.203 & 1 & 6.16 \\
\hline 0.916 & 4.246 & 1.044 & 4.1 & 0.754 & 1.215 & 1 & 6.20 \\
\hline 0.916 & 4.980 & 1.403 & 6.4 & 0.718 & 1.275 & 1 & 6.53 \\
\hline 0.918 & 4.986 & 1.419 & 6.5 & 0.715 & 1.283 & 1 & 6.54 \\
\hline 0.916 & 5.100 & 1.447 & 6.8 & 0.716 & 1.279 & 1 & 6.57 \\
\hline 0.916 & 5.602 & 1.766 & 9.1 & 0.685 & 1.338 & 1 & 6.87 \\
\hline 0.916 & 5.546 & 1.832 & 9.3 & $0: 670$ & 1.358 & 1 & 6.92 \\
\hline 0.916 & 6.540 & 2.441 & 14.6 & 0.627 & 1.462 & 1 & 7.50 \\
\hline 0.916 & 6.777 & 2.494 & 15.5 & 0.632 & 1.449 & 1 & 7.56 \\
\hline 0.918 & 6.713 & 2.541 & 15.7 & 0.621 & 1.477 & 1 & 7.60 \\
\hline 0.916 & 7.102 & 2.705 & 17.6 & 0.619 & 1.480 & 1 & 7.77 \\
\hline 0.916 & 7.321 & 2.833 & 19.0 & 0.613 & 1.494 & 1 & 7.90 \\
\hline 0.918 & 7.285 & 2.946 & 19.7 & 0.596 & 1.541 & 1 & 7.99 \\
\hline 0.916 & 7.473 & 3.042 & 20.8 & 0.593 & 1.545 & 1 & 8.09 \\
\hline 0.916 & 7.728 & 3.243 & 23.0 & 0.580 & 1.578 & 1 & 8.28 \\
\hline 0.916 & 8.077 & 3.469 & 25.7 & 0.571 & 1.605 & 1 & 8.51 \\
\hline 0.916 & 8.421 & 3.665 & 28.3 & 0.565 & 1.622 & 1 & 8.71 \\
\hline 0.916 & 8.406 & 3.766 & 29.0 & 0.552 & 1.659 & 1 & 8.79 \\
\hline 0.916 & 9.029 & 4.138 & 34.2 & 0.542 & 1.691 & 1 & 9.17 \\
\hline 0.916 & 8.978 & 4.152 & 34.1 & 0.537 & 1.704 & 1 & 9.18 \\
\hline 0.916 & 9.548 & 4.510 & 39.4 & 0.528 & 1.736 & 1 & 9.55 \\
\hline 0.916 & 9.574 & 4.528 & 39.7 & 0.527 & 1.738 & I & 9.56 \\
\hline 0.916 & 9.969 & 4.827 & 44.1 & 0.516 & 1.776 & 1 & 9.86 \\
\hline
\end{tabular}

Polyvinyl Chloride (Boltaron-Mosites)

\begin{tabular}{|c|c|c|c|c|c|c|c|}
\hline $\begin{array}{c}\rho_{o} \\
\left(\mathrm{~g} / \mathrm{cm}^{3}\right)\end{array}$ & $\begin{array}{c}u_{s} \\
(\mathrm{~km} / \mathrm{s})\end{array}$ & $\begin{array}{c}u_{p} \\
(\mathrm{~km} / \mathrm{s})\end{array}$ & $\begin{array}{c}P \\
(\mathrm{GPa})\end{array}$ & $V / V_{o}$ & $\begin{array}{c}\rho \\
\left(\mathrm{g} / \mathrm{cm}^{3}\right)\end{array}$ & std & $\begin{array}{c}u_{s s t d} \\
(\mathrm{~km} / \mathrm{s})\end{array}$ \\
\hline 1.376 & 1.930 & 0.000 & 0.0 & 1.000 & 1.376 & 40 & 0.00 \\
\hline 1.376 & 3.353 & 0.716 & 3.3 & 0.786 & 1.750 & 1 & 5.95 \\
\hline 1.376 & 3.717 & 0.942 & 4.8 & 0.746 & 1.843 & 1 & 6.16 \\
\hline 1.376 & 4.375 & 1.359 & 8.2 & 0.689 & 1.996 & 1 & 6.56 \\
\hline 1.376 & 4.397 & 1.362 & 8.2 & 0.690 & 1.993 & 1 & 6.57 \\
\hline 1.376 & 4.962 & 1.700 & 11.6 & 0.657 & 2.093 & 1 & 6.91 \\
\hline 1.376 & 5.017 & 1.760 & 12.1 & 0.649 & 2.119 & 1 & 6.97 \\
\hline 1.376 & 5.853 & 2.368 & 19.1 & 0.595 & 2.311 & 1 & 7.59 \\
\hline 1.376 & 5.907 & 2.396 & 19.5 & 0.594 & 2.315 & 1 & 7.62 \\
\hline 1.376 & 6.127 & 2.536 & 21.4 & 0.586 & 2.348 & 1 & 7.77 \\
\hline 1.376 & 6.140 & 2.563 & 21.7 & 0.583 & 2.362 & 1 & 7.79 \\
\hline 1.376 & 6.366 & 2.753 & 24.1 & 0.568 & 2.424 & 1 & 7.99 \\
\hline 1.376 & 6.734 & 3.043 & 28.2 & 0.548 & 2.510 & 1 & 8.29 \\
\hline 1.376 & 6.826 & 3.092 & 29.0 & 0.547 & 2.515 & 1 & 8.35 \\
\hline 1.376 & 7.082 & 3.258 & 31.7 & 0.540 & 2.548 & 1 & 8.53 \\
\hline 1.376 & 7.357 & 3.413 & 34.5 & 0.536 & 2.567 & 1 & 8.70 \\
\hline 1.376 & 7.417 & 3.499 & 35.7 & 0.528 & 2.605 & 1 & 8.79 \\
\hline 1.376 & 7.752 & 3.648 & 38.9 & 0.529 & 2.599 & 1 & 8.97 \\
\hline 1.366 & 7.797 & 3.676 & 39.1 & 0.529 & 2.584 & 1 & 8.99 \\
\hline 1.376 & 7.812 & 3.679 & 39.5 & 0.529 & 2.601 & 1 & 9.00 \\
\hline 1.376 & 7.959 & 3.889 & 42.6 & 0.511 & 2.690 & 1 & 9.21 \\
\hline 1.376 & 8.148 & 4.081 & 45.7 & 0.499 & 2.756 & 1 & 9.41 \\
\hline 1.376 & 8.368 & 4.155 & 47.8 & 0.503 & 2.733 & 1 & 9.51 \\
\hline 1.376 & 8.427 & 4.228 & 49.0 & 0.498 & 2.761 & 1 & 9.58 \\
\hline 1.376 & 8.818 & 4.382 & 53.2 & 0.503 & 2.735 & 1 & 9.77 \\
\hline 1.376 & 8.736 & 4.409 & 53.0 & 0.495 & 2.778 & 1 & 9.79 \\
\hline 1.376 & 8.699 & 4.416 & 52.9 & 0.492 & 2.794 & 1 & 9.79 \\
\hline 1.376 & 8.640 & 4.420 & 52.5 & 0.488 & 2.817 & 1 & 9.78 \\
\hline 1.376 & 9.100 & 4.564 & 57.2 & 0.498 & 2.761 & 1 & 9.98 \\
\hline 1.376 & 9.062 & 4.614 & 57.5 & 0.491 & 2.803 & 1 & 10.02 \\
\hline 1.376 & 9.592 & 4.907 & 64.8 & 0.488 & 2.817 & 1 & 10.36 \\
\hline
\end{tabular}


Polyvinylidene Fluoride (Kynar-Pennsalt)

\begin{tabular}{rccccrrr}
\hline \hline $\begin{array}{c}\rho_{o} \\
\left(\mathrm{~g} / \mathrm{cm}^{3}\right)\end{array}$ & $\begin{array}{c}u_{s} \\
(\mathrm{~km} / \mathrm{s})\end{array}$ & $\begin{array}{c}u_{p} \\
(\mathrm{~km} / \mathrm{s})\end{array}$ & $\begin{array}{c}P \\
(\mathrm{GPa})\end{array}$ & $V / V_{o}$ & $\begin{array}{c}\rho \\
\left(\mathrm{g} / \mathrm{cm}^{3}\right)\end{array}$ & std & $\begin{array}{c}u_{\text {sstd }} \\
(\mathrm{km} / \mathrm{s})\end{array}$ \\
\hline 1.768 & 1.850 & 0.000 & 0.0 & 1.000 & 1.768 & 40 & 0.00 \\
1.766 & 3.562 & 0.642 & 4.0 & 0.820 & 2.154 & 1 & 5.93 \\
1.766 & 3.604 & 0.662 & 4.2 & 0.816 & 2.163 & 1 & 5.95 \\
1.766 & 3.897 & 0.816 & 5.6 & 0.791 & 2.234 & 1 & 6.11 \\
1.766 & 3.963 & 0.869 & 6.1 & 0.781 & 2.262 & $\mathbf{1}$ & 6.16 \\
1.766 & 4.573 & 1.255 & 10.1 & 0.726 & 2.434 & 1 & 6.56 \\
1.768 & 5.136 & 1.569 & 14.2 & 0.695 & 2.546 & 1 & 6.91 \\
1.768 & 5.167 & 1.627 & 14.9 & 0.685 & 2.580 & 1 & 6.97 \\
1.766 & 6.057 & 2.192 & 23.4 & 0.638 & 2.767 & 1 & 7.60 \\
1.766 & 6.042 & 2.215 & 23.6 & 0.633 & 2.788 & 1 & 7.62 \\
1.766 & 6.279 & 2.341 & 26.0 & 0.627 & 2.816 & 1 & 7.77 \\
1.766 & 6.355 & 2.359 & 26.5 & 0.629 & 2.809 & 1 & 7.79 \\
1.766 & 6.569 & 2.536 & 29.4 & 0.614 & 2.877 & 1 & 7.99 \\
1.766 & 6.891 & 2.809 & 34.2 & 0.592 & 2.982 & 1 & 8.29 \\
1.766 & 6.910 & 2.864 & 34.9 & 0.586 & 3.016 & 1 & 8.35 \\
1.766 & 7.264 & 3.004 & 38.5 & 0.586 & 3.011 & 1 & 8.53 \\
1.766 & 7.400 & 3.164 & 41.4 & 0.572 & 3.085 & 1 & 8.70 \\
1.766 & 7.479 & 3.242 & 42.8 & 0.567 & 3.117 & 1 & 8.79 \\
1.766 & 7.785 & 3.419 & 47.0 & 0.561 & 3.149 & 1 & 9.00 \\
1.766 & 8.152 & 3.789 & 54.6 & 0.535 & 3.300 & 1 & 9.41 \\
1.768 & 8.479 & 3.916 & 58.7 & 0.538 & 3.285 & 1 & 9.58 \\
1.768 & 8.730 & 4.091 & 63.1 & 0.531 & 3.327 & 1 & 9.79 \\
1.766 & 8.652 & 4.101 & 62.7 & 0.526 & 3.358 & 1 & 9.78 \\
1.768 & 8.931 & 4.238 & 66.9 & 0.525 & 3.365 & 1 & 9.96 \\
1.766 & 9.055 & 4.240 & 67.8 & 0.532 & 3.321 & 1 & 9.98 \\
1.766 & 9.412 & 4.578 & 76.1 & 0.514 & 3.439 & 1 & 10.36 \\
1.768 & 9.208 & 4.578 & 74.5 & 0.503 & 3.516 & 1 & 10.33 \\
\hline \hline
\end{tabular}

Polychlorotrifluoroethylene (Kel F-Fluorocarbon)

\begin{tabular}{rccccrrr}
\hline $\begin{array}{c}\rho_{o} \\
\left(\mathrm{~g} / \mathrm{cm}^{3}\right)\end{array}$ & $\begin{array}{c}u_{s} \\
(\mathrm{~km} / \mathrm{s})\end{array}$ & $\begin{array}{c}u_{p} \\
(\mathrm{~km} / \mathrm{s})\end{array}$ & $\begin{array}{c}P \\
(\mathrm{GPa})\end{array}$ & $V / V_{o}$ & $\begin{array}{c}\rho \\
\left(\mathrm{g} / \mathrm{cm}^{3}\right)\end{array}$ & std & $\begin{array}{r}u_{s s t d} \\
(\mathrm{~km} / \mathrm{s})\end{array}$ \\
\hline 2.133 & 1.501 & 0.000 & 0.0 & 1.000 & 2.133 & 40 & 0.00 \\
2.131 & 3.132 & 0.604 & 4.0 & 0.807 & 2.640 & 1 & 5.90 \\
2.131 & 3.152 & 0.689 & 4.6 & 0.781 & 2.728 & 1 & 5.98 \\
2.133 & 3.485 & 0.869 & 6.5 & 0.751 & 2.841 & 1 & 6.17 \\
2.133 & 3.465 & 0.905 & 6.7 & 0.739 & 2.887 & 1 & 6.21 \\
2.134 & 4.113 & 1.221 & 10.7 & 0.703 & 3.035 & 1 & 6.56 \\
2.132 & 4.113 & 1.247 & 10.9 & 0.697 & 3.059 & 1 & 6.58 \\
2.134 & 4.669 & 1.546 & 15.4 & 0.669 & 3.190 & 1 & 6.93 \\
2.134 & 4.658 & 1.565 & 15.6 & 0.664 & 3.214 & 1 & 6.95 \\
2.131 & 5.462 & 2.052 & 23.9 & 0.624 & 3.413 & 1 & 7.52 \\
2.133 & 5.517 & 2.088 & 24.6 & 0.622 & 3.432 & 1 & 7.56 \\
2.134 & 5.796 & 2.295 & 28.4 & 0.604 & 3.533 & 1 & 7.80 \\
2.132 & 5.974 & 2.343 & 29.8 & 0.608 & 3.508 & 1 & 7.87 \\
2.132 & 6.483 & 2.745 & 37.9 & 0.577 & 3.698 & 1 & 8.34 \\
2.134 & 6.779 & 2.841 & 41.1 & 0.581 & 3.674 & 10 & 6.69 \\
2.133 & 6.913 & 2.900 & 42.8 & 0.581 & 3.674 & 1 & 8.56 \\
2.132 & 6.907 & 2.912 & 42.9 & 0.578 & 3.686 & 10 & 6.77 \\
2.134 & 6.959 & 2.934 & 43.6 & 0.578 & 3.689 & 1 & 8.60 \\
2.133 & 7.156 & 3.064 & 46.8 & 0.572 & 3.730 & 1 & 8.76 \\
2.134 & 7.484 & 3.305 & 52.8 & 0.558 & 3.821 & 1 & 9.05 \\
2.132 & 7.971 & 3.652 & 62.1 & 0.542 & 3.935 & 1 & 9.48 \\
2.132 & 8.590 & 4.017 & 73.6 & 0.532 & 4.005 & 1 & 9.94 \\
2.132 & 8.658 & 4.064 & 75.0 & 0.531 & 4.018 & 1 & 9.99 \\
2.132 & 8.631 & 4.068 & 74.9 & 0.529 & 4.033 & 1 & 9.99 \\
\hline \hline
\end{tabular}


Polytetrafluoroethylene (Teflon-Dupont)

\begin{tabular}{|c|c|c|c|c|c|c|c|}
\hline $\begin{array}{c}\rho_{o} \\
\left(\mathrm{~g} / \mathrm{cm}^{3}\right)\end{array}$ & $\begin{array}{c}u_{s} \\
(\mathrm{~km} / \mathrm{s})\end{array}$ & $\begin{array}{c}u_{p} \\
(\mathrm{~km} / \mathrm{s}) \\
\end{array}$ & $\begin{array}{c}P \\
(\mathrm{GPa}) \\
\end{array}$ & $V / V_{o}$ & $\begin{array}{c}\rho \\
\left(\mathrm{g} / \mathrm{cm}^{3}\right) \\
\end{array}$ & std & $\begin{array}{c}u_{s s t d} \\
(\mathrm{~km} / \mathrm{s})\end{array}$ \\
\hline 2.151 & 1.139 & 0.000 & 0.0 & 1.000 & 2.151 & 40 & 0.00 \\
\hline 2.152 & 2.889 & 0.662 & 4.1 & 0.771 & 2.792 & 1 & 5.94 \\
\hline 2.153 & 2.822 & 0.709 & 4.3 & 0.749 & 2.875 & 1 & 5.98 \\
\hline 2.152 & 3.255 & 0.847 & 5.9 & 0.740 & 2.909 & 1 & 6.14 \\
\hline 2.153 & 3.235 & 0.921 & 6.4 & 0.715 & 3.010 & 1 & 6.21 \\
\hline 2.152 & 3.707 & 1.087 & 8.7 & 0.707 & 3.045 & 10 & 4.92 \\
\hline 2.152 & 3.913 & 1.206 & 10.2 & 0.692 & 3.110 & 1 & 6.53 \\
\hline 2.153 & 3.907 & 1.263 & 10.6 & 0.677 & 3.181 & 1 & 6.58 \\
\hline 2.152 & 4.512 & 1.540 & 14.9 & 0.659 & 3.267 & 1 & 6.91 \\
\hline 2.153 & 4.518 & 1.550 & 15.1 & 0.657 & 3.278 & 1 & 6.92 \\
\hline 2.153 & 4.457 & 1.569 & 15.1 & 0.648 & 3.323 & 1 & 6.94 \\
\hline 2.152 & 4.645 & 1.687 & 16.9 & 0.637 & 3.379 & 10 & 5.50 \\
\hline 2.153 & 4.903 & 1.788 & 18.9 & 0.635 & 3.389 & 10 & 5.61 \\
\hline 2.153 & 5.453 & 2.046 & 24.0 & 0.625 & 3.446 & 1 & 7.52 \\
\hline 2.152 & 5.430 & 2.072 & 24.2 & 0.618 & 3.480 & 1 & 7.54 \\
\hline 2.153 & 5.516 & 2.109 & 25.1 & 0.618 & 3.486 & 1 & 7.59 \\
\hline 2.153 & 5.717 & 2.242 & 27.6 & 0.608 & 3.542 & 1 & 7.75 \\
\hline 2.154 & 5.729 & 2.289 & 28.2 & 0.600 & 3.587 & 10 & 6.12 \\
\hline 2.152 & 5.770 & 2.321 & 28.8 & 0.598 & 3.600 & 10 & 6.15 \\
\hline 2.156 & 5.895 & 2.378 & 30.2 & 0.597 & 3.614 & 1 & 7.91 \\
\hline 2.154 & 5.927 & 2.409 & 30.7 & 0.594 & 3.629 & 10 & 6.24 \\
\hline 2.152 & 5.990 & 2.410 & 31.1 & 0.598 & 3.601 & 1 & 7.95 \\
\hline 2.153 & 6.036 & 2.425 & 31.5 & 0.598 & 3.599 & 1 & 7.97 \\
\hline 2.153 & 6.291 & 2.606 & 35.3 & 0.586 & 3.675 & 1 & 8.19 \\
\hline 2.152 & 6.379 & 2.614 & 35.9 & 0.590 & 3.646 & 10 & 6.46 \\
\hline 2.152 & 6.655 & 2.748 & 39.4 & 0.587 & 3.665 & 1 & 8.38 \\
\hline 2.155 & 6.551 & 2.856 & 40.3 & 0.564 & 3.820 & 10 & 6.69 \\
\hline 2.152 & 6.723 & 2.897 & 41.9 & 0.569 & 3.781 & 10 & 6.75 \\
\hline 2.149 & 6.751 & 2.921 & 42.4 & 0.567 & 3.788 & 10 & 6.77 \\
\hline 2.152 & 7.084 & 3.062 & 46.7 & 0.568 & 3.790 & 1 & 8.76 \\
\hline 2.152 & 7.377 & 3.261 & 51.8 & 0.558 & 3.857 & 10 & 7.13 \\
\hline 2.152 & 7.625 & 3.377 & 55.4 & 0.557 & 3.863 & 1 & 9.16 \\
\hline 2.152 & 7.673 & 3.451 & 57.0 & 0.550 & 3.911 & 1 & 9.24 \\
\hline 2.152 & 7.910 & 3.508 & 59.7 & 0.557 & 3.867 & 1 & 9.33 \\
\hline 2.152 & 7.848 & 3.565 & 60.2 & 0.546 & 3.943 & 10 & 7.45 \\
\hline 2.152 & 8.003 & 3.711 & 63.9 & 0.536 & 4.013 & 10 & 7.60 \\
\hline 2.151 & 8.040 & 3.748 & 64.8 & 0.534 & 4.030 & 10 & 7.64 \\
\hline 2.152 & 8.390 & 3.818 & 68.9 & 0.545 & 3.949 & 1 & 9.72 \\
\hline 2.152 & 8.575 & 3.987 & 73.6 & 0.535 & 4.022 & 1 & 9.92 \\
\hline 2.152 & 8.664 & 4.008 & 74.7 & 0.537 & 4.005 & 1 & 9.95 \\
\hline 2.152 & 8.602 & 4.050 & 75.0 & 0.529 & 4.067 & 1 & 9.98 \\
\hline 2.152 & 8.604 & 4.078 & 75.5 & 0.526 & 4.091 & 1 & 10.01 \\
\hline 2.152 & 9.153 & 4.375 & 86.2 & 0.522 & 4.123 & 1 & 10.40 \\
\hline
\end{tabular}


Polypropylene (Avisun)

\begin{tabular}{|c|c|c|c|c|c|c|c|}
\hline $\begin{array}{c}\rho_{o} \\
\left(\mathrm{~g} / \mathrm{cm}^{3}\right)\end{array}$ & $\begin{array}{c}u_{s} \\
(\mathrm{~km} / \mathrm{s})\end{array}$ & $\begin{array}{c}u_{p} \\
(\mathrm{~km} / \mathrm{s})\end{array}$ & $\begin{array}{c}P \\
(\mathrm{GPa})\end{array}$ & $V / V_{o}$ & $\begin{array}{c}\rho \\
\left(\mathrm{g} / \mathrm{cm}^{3}\right)\end{array}$ & std & $\begin{array}{c}u_{s s t d} \\
(\mathrm{~km} / \mathrm{s})\end{array}$ \\
\hline 0.904 & 2.140 & 0.000 & 0.0 & 1.000 & 0.904 & 40 & 0.00 \\
\hline 0.904 & 3.939 & 0.730 & 2.6 & 0.815 & 1.110 & 1 & 5.93 \\
\hline 0.904 & 3.983 & 0.753 & 2.7 & 0.811 & 1.115 & 1 & 5.95 \\
\hline 0.904 & 4.295 & 0.933 & 3.6 & 0.783 & 1.155 & 1 & 6.11 \\
\hline 0.904 & 4.332 & 0.997 & 3.9 & 0.770 & 1.174 & 1 & 6.16 \\
\hline 0.904 & 5.019 & 1.447 & 6.6 & 0.712 & 1.270 & 1 & 6.56 \\
\hline 0.904 & 5.509 & 1.846 & 9.2 & 0.665 & 1.360 & 1 & 6.92 \\
\hline 0.904 & 5.678 & 1.887 & 9.7 & 0.668 & 1.354 & 1 & 6.97 \\
\hline 0.904 & 6.710 & 2.538 & 15.4 & 0.622 & 1.454 & 1 & 7.59 \\
\hline 0.904 & 6.702 & 2.575 & 15.6 & 0.616 & 1.468 & 1 & 7.62 \\
\hline 0.904 & 6.979 & 2.725 & 17.2 & 0.610 & 1.483 & 1 & 7.77 \\
\hline 0.904 & 7.193 & 2.941 & 19.1 & 0.591 & 1.529 & 1 & 7.97 \\
\hline 0.904 & 7.567 & 3.157 & 21.6 & 0.583 & 1.551 & 1 & 8.19 \\
\hline 0.903 & 7.646 & 3.216 & 22.2 & 0.579 & 1.559 & 1 & 8.24 \\
\hline 0.904 & 7.673 & 3.273 & 22.7 & 0.573 & 1.576 & 1 & 8.29 \\
\hline 0.904 & 7.706 & 3.334 & 23.2 & 0.567 & 1.593 & 1 & 8.35 \\
\hline 0.903 & 8.340 & 3.712 & 28.0 & 0.555 & 1.627 & 1 & 8.73 \\
\hline 0.904 & 8.448 & 3.769 & 28.8 & 0.554 & 1.632 & 1 & 8.79 \\
\hline 0.904 & 8.628 & 3.955 & 30.8 & 0.542 & 1.669 & 1 & 8.97 \\
\hline 0.904 & 8.802 & 3.977 & 31.6 & 0.548 & 1.649 & 1 & 9.00 \\
\hline 0.904 & 9.269 & 4.400 & 36.9 & 0.525 & 1.721 & 1 & 9.41 \\
\hline 0.904 & 9.570 & 4.563 & 39.5 & 0.523 & 1.728 & 1 & 9.58 \\
\hline 0.904 & 9.831 & 4.771 & 42.4 & 0.515 & 1.757 & 1 & 9.79 \\
\hline 0.904 & 9.704 & 4.783 & 42.0 & 0.507 & 1.783 & 1 & 9.78 \\
\hline 0.904 & 10.040 & 4.967 & 45.1 & 0.505 & 1.789 & 1 & 9.98 \\
\hline 0.904 & 9.832 & 4.970 & 44.2 & 0.494 & 1.828 & 1 & 9.96 \\
\hline 0.903 & 10.163 & 5.000 & 45.9 & 0.508 & 1.778 & 1 & 10.02 \\
\hline 0.904 & 10.440 & 5.327 & 50.3 & 0.490 & 1.846 & $\mathbf{I}$ & 10.33 \\
\hline 0.904 & 10.613 & 5.339 & 51.2 & 0.497 & 1.819 & 1 & 10.36 \\
\hline 0.903 & 10.411 & 5.418 & 50.9 & 0.480 & 1.883 & $\mathbf{1}$ & 10.40 \\
\hline 0.903 & 10.507 & 5.435 & 51.6 & 0.483 & 1.871 & 1 & 10.43 \\
\hline
\end{tabular}


Poly-(4-methyl-1-pentene) (DX816-Imperial, TPX)

\begin{tabular}{|c|c|c|c|c|c|c|c|}
\hline $\begin{array}{c}\rho_{\circ} \\
\left(\mathrm{g} / \mathrm{cm}^{3}\right)\end{array}$ & $\begin{array}{c}u_{s} \\
(\mathrm{~km} / \mathrm{s})\end{array}$ & $\begin{array}{c}u_{p} \\
(\mathrm{~km} / \mathrm{s})\end{array}$ & $\begin{array}{c}P \\
(\mathrm{GPa})\end{array}$ & $V / V_{o}$ & $\begin{array}{c}\rho \\
\left(\mathrm{g} / \mathrm{cm}^{3}\right)\end{array}$ & std & $\begin{array}{c}u_{s s t d} \\
(\mathrm{~km} / \mathrm{s})\end{array}$ \\
\hline 0.829 & 1.806 & 0.000 & 0.0 & 1.000 & 0.829 & 40 & 0.00 \\
\hline 0.830 & 2.798 & 0.489 & 1.1 & 0.825 & 1.005 & 10 & 4.33 \\
\hline 0.830 & 3.019 & 0.594 & 1.5 & 0.803 & 1.033 & 10 & 4.41 \\
\hline 0.830 & 3.706 & 1.009 & 3.1 & 0.728 & 1.141 & 10 & 4.75 \\
\hline 0.829 & 3.738 & 1.013 & 3.1 & 0.729 & 1.137 & 1 & 6.14 \\
\hline 0.830 & 4.032 & 1.225 & 4.1 & 0.696 & 1.192 & 10 & 4.93 \\
\hline 0.830 & 4.464 & 1.459 & 5.4 & 0.673 & 1.233 & 1 & 6.53 \\
\hline 0.829 & 4.446 & 1.461 & 5.4 & 0.671 & 1.235 & 1 & 6.53 \\
\hline 0.830 & 4.927 & 1.766 & 7.2 & 0.641 & 1.294 & 10 & 5.39 \\
\hline 0.831 & 4.951 & 1.804 & 7.4 & 0.636 & 1.307 & 10 & 5.42 \\
\hline 0.831 & 4.910 & 1.805 & 7.4 & 0.632 & 1.314 & 10 & 5.42 \\
\hline 0.830 & 4.918 & 1.816 & 7.4 & 0.631 & 1.316 & 10 & 5.43 \\
\hline 0.831 & 4.902 & 1.816 & 7.4 & 0.629 & 1.320 & 10 & 5.43 \\
\hline 0.831 & 4.947 & 1.859 & 7.6 & 0.624 & 1.331 & 10 & 5.47 \\
\hline 0.831 & 4.939 & 1.860 & 7.6 & 0.624 & 1.333 & 10 & 5.47 \\
\hline 0.829 & 5.073 & 1.873 & 7.9 & 0.631 & 1.314 & 1 & 6.89 \\
\hline 0.830 & 5.146 & 1.912 & 8.2 & 0.628 & 1.321 & 1 & 6.93 \\
\hline 0.830 & 5.167 & 1.951 & 8.4 & 0.622 & 1.334 & 1 & 6.96 \\
\hline 0.830 & 5.239 & 1.976 & 8.6 & 0.623 & 1.333 & 10 & 5.57 \\
\hline 0.829 & 6.199 & 2.546 & 13.1 & 0.589 & 1.407 & 1 & 7.52 \\
\hline 0.827 & 6.242 & 2.595 & 13.4 & 0.584 & 1.415 & 1 & 7.56 \\
\hline 0.829 & 6.262 & 2.662 & 13.8 & 0.575 & 1.442 & 1 & 7.62 \\
\hline 0.830 & 6.451 & 2.772 & 14.8 & 0.570 & 1.456 & 10 & 6.26 \\
\hline 0.831 & 6.419 & 2.773 & 14.8 & 0.568 & 1.462 & 10 & 6.26 \\
\hline 0.827 & 6.666 & 2.806 & 15.5 & 0.579 & 1.428 & 1 & 7.77 \\
\hline 0.830 & 6.634 & 2.904 & 16.0 & 0.562 & 1.476 & 10 & 6.38 \\
\hline 0.827 & 6.808 & 2.945 & 16.6 & 0.567 & 1.458 & 1 & 7.90 \\
\hline 0.831 & 7.078 & 3.248 & 19.1 & 0.541 & 1.536 & 10 & 6.67 \\
\hline 0.831 & 7.078 & 3.248 & 19.1 & 0.541 & 1.536 & 10 & 6.67 \\
\hline 0.830 & 7.094 & 3.250 & 19.1 & 0.542 & 1.532 & 10 & 6.68 \\
\hline 0.829 & 7.160 & 3.258 & 19.3 & 0.545 & 1.521 & 1 & 8.19 \\
\hline 0.827 & 7.313 & 3.359 & 20.3 & 0.541 & 1.530 & 1 & 8.28 \\
\hline 0.829 & 7.547 & 3.464 & 21.7 & 0.541 & 1.532 & 1 & 8.39 \\
\hline 0.830 & 7.553 & 3.562 & 22.3 & 0.528 & 1.571 & 10 & 6.95 \\
\hline 0.829 & 7.779 & 3.620 & 23.3 & 0.535 & 1.551 & 1 & 8.54 \\
\hline 0.827 & 7.925 & 3.777 & 24.8 & 0.523 & 1.580 & 1 & 8.68 \\
\hline 0.829 & 8.038 & 3.798 & 25.3 & 0.527 & 1.572 & 1 & 8.72 \\
\hline 0.829 & 8.428 & 3.952 & 27.6 & 0.531 & 1.561 & 1 & 8.88 \\
\hline 0.830 & 8.344 & 4.129 & 28.6 & 0.505 & 1.643 & 10 & 7.45 \\
\hline 0.830 & 8.657 & 4.261 & 30.6 & 0.508 & 1.635 & 10 & 7.57 \\
\hline 0.829 & 8.698 & 4.275 & 30.8 & 0.509 & 1.630 & 1 & 9.18 \\
\hline 0.830 & 8.721 & 4.310 & 31.2 & 0.506 & 1.641 & 1 & 9.21 \\
\hline 0.830 & 8.800 & 4.474 & 32.7 & 0.492 & 1.688 & 10 & 7.75 \\
\hline 0.829 & 9.080 & 4.516 & 34.0 & 0.503 & 1.649 & 1 & 9.42 \\
\hline 0.829 & 9.329 & 4.575 & 35.4 & 0.510 & 1.627 & 1 & 9.49 \\
\hline 0.829 & 9.575 & 4.602 & 36.5 & 0.519 & 1.596 & 1 & 9.54 \\
\hline 0.830 & 9.309 & 4.801 & 37.1 & 0.484 & 1.714 & 1 & 9.68 \\
\hline 0.830 & 9.606 & 4.836 & 38.6 & 0.497 & 1.672 & 1 & 9.74 \\
\hline 0.830 & 9.715 & 4.994 & 40.3 & 0.486 & 1.708 & 1 & 9.88 \\
\hline 0.830 & 9.426 & 5.013 & 39.2 & 0.468 & 1.773 & 10 & 8.22 \\
\hline 0.830 & 9.777 & 5.040 & 40.9 & 0.484 & 1.713 & 1 & 9.93 \\
\hline 0.830 & 10.328 & 5.484 & 47.9 & 0.469 & 1.770 & 1 & 10.36 \\
\hline
\end{tabular}


Polyamide (Nylon 6/6-Polypenco 101)

\begin{tabular}{|c|c|c|c|c|c|c|c|}
\hline $\begin{array}{c}\rho_{o} \\
\left(\mathrm{~g} / \mathrm{cm}^{3}\right)\end{array}$ & $\begin{array}{c}u_{s} \\
(\mathrm{~km} / \mathrm{s})\end{array}$ & $\begin{array}{c}u_{p} \\
(\mathrm{~km} / \mathrm{s})\end{array}$ & $\begin{array}{c}P \\
(\mathrm{GPa})\end{array}$ & $V / V_{o}$ & $\begin{array}{c}\rho \\
\left(\mathrm{g} / \mathrm{cm}^{3}\right) \\
\end{array}$ & std & $\begin{array}{c}u_{\text {sstd }} \\
(\mathrm{km} / \mathrm{s})\end{array}$ \\
\hline 1.140 & 2.208 & 0.000 & 0.0 & 1.000 & 1.140 & 40 & 0.00 \\
\hline 1.140 & 3.938 & 0.762 & 3.4 & 0.806 & 1.414 & 1 & 5.98 \\
\hline 1.139 & 4.334 & 1.017 & 5.0 & 0.765 & 1.488 & 1 & 6.22 \\
\hline 1.140 & 4.944 & 1.348 & 7.6 & 0.727 & 1.568 & 1 & 6.54 \\
\hline 1.150 & 5.124 & 1.374 & 8.1 & 0.732 & 1.571 & 1 & 6.57 \\
\hline 1.150 & 5.689 & 1.728 & 11.3 & 0.696 & 1.652 & 1 & 6.92 \\
\hline 1.150 & 5.642 & 1.738 & 11.3 & 0.692 & 1.662 & 1 & 6.92 \\
\hline 1.140 & 5.540 & 1.748 & 11.0 & 0.684 & 1.666 & 1 & 6.92 \\
\hline 1.140 & 5.678 & 1.800 & 11.6 & 0.683 & 1.669 & 10 & 5.50 \\
\hline 1.150 & 6.691 & 2.366 & 18.2 & 0.646 & 1.779 & 1 & 7.56 \\
\hline 1.150 & 6.805 & 2.386 & 18.7 & 0.649 & 1.771 & 1 & 7.59 \\
\hline 1.150 & 6.870 & 2.555 & 20.2 & 0.628 & 1.831 & 1 & 7.75 \\
\hline 1.150 & 7.022 & 2.565 & 20.7 & 0.635 & 1.812 & 1 & 7.77 \\
\hline 1.150 & 7.147 & 2.694 & 22.1 & 0.623 & 1.846 & 1 & 7.90 \\
\hline 1.150 & 7.140 & 2.778 & 22.8 & 0.611 & 1.883 & 1 & 7.97 \\
\hline 1.140 & 7.134 & 2.807 & 22.8 & 0.606 & 1.880 & 10 & 6.41 \\
\hline 1.140 & 7.331 & 2.926 & 24.5 & 0.601 & 1.897 & 1 & 8.12 \\
\hline 1.150 & 7.548 & 2.976 & 25.8 & 0.606 & 1.899 & 1 & 8.19 \\
\hline 1.150 & 7.545 & 3.083 & 26.8 & 0.591 & 1.945 & 1 & 8.28 \\
\hline 1.150 & 7.831 & 3.302 & 29.7 & 0.578 & 1.988 & 1 & 8.51 \\
\hline 1.140 & 8.078 & 3.462 & 31.9 & 0.571 & 1.995 & 10 & 7.02 \\
\hline 1.150 & 8.341 & 3.777 & 36.2 & 0.547 & 2.102 & 1 & 8.98 \\
\hline 1.140 & 8.297 & 3.789 & 35.8 & 0.543 & 2.098 & 1 & 8.98 \\
\hline 1.150 & 8.599 & 4.027 & 39.8 & 0.532 & 2.163 & 1 & 9.24 \\
\hline 1.150 & 8.739 & 4.113 & 41.3 & 0.529 & 2.173 & 1 & 9.33 \\
\hline 1.140 & 9.041 & 4.312 & 44.4 & 0.523 & 2.179 & 1 & 9.53 \\
\hline 1.150 & 9.481 & 4.675 & 51.0 & 0.507 & 2.269 & 1 & 9.92 \\
\hline 1.150 & 9.528 & 4.709 & 51.6 & 0.506 & 2.274 & 1 & 9.95 \\
\hline 1.150 & 9.453 & 4.755 & 51.7 & 0.497 & 2.314 & 1 & 9.98 \\
\hline 1.150 & 9.436 & 4.790 & 52.0 & 0.492 & 2.336 & 1 & 10.01 \\
\hline 1.140 & 9.580 & 4.795 & 52.4 & 0.500 & 2.282 & 1 & 10.03 \\
\hline 1.150 & 10.032 & 5.144 & 59.4 & 0.487 & 2.360 & 1 & 10.40 \\
\hline
\end{tabular}


Polymethylmethacrylate (Rohm and Hass 11, Plexiglas)

\begin{tabular}{|c|c|c|c|c|c|c|c|}
\hline $\begin{array}{c}\rho_{o} \\
\left(\mathrm{~g} / \mathrm{cm}^{3}\right)\end{array}$ & $\begin{array}{c}u_{s} \\
(\mathrm{~km} / \mathrm{s})\end{array}$ & $\begin{array}{c}u_{p} \\
(\mathrm{~km} / \mathrm{s})\end{array}$ & $\begin{array}{c}P \\
(\mathrm{GPa})\end{array}$ & $V / V_{o}$ & $\begin{array}{c}\rho \\
\left(\mathrm{g} / \mathrm{cm}^{3}\right)\end{array}$ & std & $\begin{array}{c}u_{s s t d} \\
(\mathrm{~km} / \mathrm{s})\end{array}$ \\
\hline 1.189 & 2.229 & 0.000 & 0.0 & 1.000 & 1.189 & 40 & 0.00 \\
\hline 1.191 & 3.345 & 0.375 & 1.5 & 0.888 & 1.342 & 1 & 5.64 \\
\hline 1.187 & 3.350 & 0.469 & 1.9 & 0.860 & 1.380 & 10 & 4.33 \\
\hline 1.190 & 3.389 & 0.530 & 2.1 & 0.843 & 1.411 & 1 & 5.78 \\
\hline 1.186 & 3.446 & 0.546 & 2.2 & 0.842 & 1.409 & 1 & 5.79 \\
\hline 1.187 & 3.501 & 0.570 & 2.4 & 0.837 & 1.418 & 10 & 4.41 \\
\hline 1.186 & 3.612 & 0.700 & 3.0 & 0.806 & 1.471 & 1 & 5.92 \\
\hline 1.186 & 3.709 & 0.706 & 3.1 & 0.810 & 1.465 & 1 & 5.93 \\
\hline 1.186 & 3.702 & 0.717 & 3.1 & 0.806 & 1.471 & 1 & 5.94 \\
\hline 1.190 & 3.604 & 0.719 & 3.1 & 0.801 & 1.486 & 1 & 5.94 \\
\hline 1.189 & 3.757 & 0.756 & 3.4 & 0.799 & 1.489 & 1 & 5.98 \\
\hline 1.186 & 3.718 & 0.765 & 3.4 & 0.794 & 1.493 & 1 & 5.98 \\
\hline 1.189 & 3.741 & 0.788 & 3.5 & 0.789 & 1.506 & 2 & 5.76 \\
\hline 1.186 & 3.836 & 0.841 & 3.8 & 0.781 & 1.519 & 1 & 6.05 \\
\hline 1.186 & 3.908 & 0.918 & 4.3 & 0.765 & 1.550 & 1 & 6.12 \\
\hline 1.187 & 4.029 & 0.941 & 4.5 & 0.766 & 1.549 & 1 & 6.15 \\
\hline 1.187 & 4.008 & 0.942 & 4.5 & 0.765 & 1.552 & 1 & 6.15 \\
\hline 1.187 & 4.014 & 0.966 & 4.6 & 0.759 & 1.563 & 1 & 6.17 \\
\hline 1.187 & 4.070 & 0.968 & 4.7 & 0.762 & 1.557 & 10 & 4.75 \\
\hline 1.186 & 4.050 & 0.980 & 4.7 & 0.578 & 1.565 & 1 & 6.18 \\
\hline 1.187 & 4.392 & 1.172 & 6.1 & 0.733 & 1.619 & 10 & 4.93 \\
\hline 1.187 & 4.453 & 1.210 & 6.4 & 0.728 & 1.630 & 1 & 6.40 \\
\hline 1.187 & 4.519 & 1.267 & 6.8 & 0.720 & 1.649 & 1 & 6.45 \\
\hline 1.189 & 4.667 & 1.344 & 7.5 & 0.712 & 1.670 & 1 & 6.53 \\
\hline 1.187 & 4.573 & 1.346 & 7.3 & 0.706 & 1.682 & 1 & 6.52 \\
\hline 1.187 & 4.688 & 1.367 & 7.6 & 0.708 & 1.676 & 1 & 6.55 \\
\hline 1.187 & 4.683 & 1.367 & 7.6 & 0.708 & 1.677 & 1 & 6.55 \\
\hline 1.184 & 4.589 & 1.371 & 7.5 & 0.701 & 1.689 & 1 & 6.55 \\
\hline 1.187 & 4.701 & 1.419 & 7.9 & 0.698 & 1.700 & 1 & 6.59 \\
\hline 1.189 & 4.704 & 1.461 & 8.2 & 0.689 & 1.725 & 2 & 6.43 \\
\hline 1.187 & 5.143 & 1.672 & 10.2 & 0.675 & 1.759 & 1 & 6.84 \\
\hline 1.187 & 5.181 & 1.689 & 10.4 & 0.674 & 1.761 & 10 & 5.39 \\
\hline 1.187 & 5.230 & 1.723 & 10.7 & 0.670 & 1.770 & 10 & 5.42 \\
\hline 1.187 & 5.275 & 1.732 & 10.8 & 0.672 & 1.767 & 10 & 5.43 \\
\hline 1.187 & 5.136 & 1.744 & 10.7 & 0.660 & 1.800 & 1 & 6.91 \\
\hline 1.187 & 5.306 & 1.759 & 11.1 & 0.669 & 1.775 & 1 & 6.93 \\
\hline 1.185 & 5.291 & 1.774 & 11.1 & 0.665 & 1.783 & 10 & 5.47 \\
\hline 1.183 & 5.292 & 1.775 & 11.1 & 0.665 & 1.780 & 10 & 5.47 \\
\hline 1.189 & 5.303 & 1.866 & 11.8 & 0.648 & 1.834 & 2 & 6.86 \\
\hline 1.187 & 5.507 & 1.866 & 12.3 & 0.658 & 1.805 & 10 & 5.57 \\
\hline 1.189 & 6.241 & 2.367 & 17.6 & 0.621 & 1.916 & 1 & 7.55 \\
\hline 1.189 & 6.324 & 2.387 & 17.9 & 0.623 & 1.910 & 2 & 7.43 \\
\hline 1.189 & 6.324 & 2.478 & 18.6 & 0.608 & 1.955 & 2 & 7.52 \\
\hline 1.187 & 6.641 & 2.639 & 20.8 & 0.603 & 1.970 & 10 & 6.26 \\
\hline 1.187 & 6.641 & 2.639 & 20.8 & 0.603 & 1.970 & 10 & 6.26 \\
\hline 1.187 & 6.621 & 2.640 & 20.7 & 0.601 & 1.974 & 10 & 6.26 \\
\hline 1.187 & 6.790 & 2.766 & 22.3 & 0.593 & 2.003 & 10 & 6.38 \\
\hline 1.187 & 6.938 & 2.872 & 23.7 & 0.586 & 2.025 & 10 & 6.47 \\
\hline 1.186 & 7.182 & 3.052 & 26.0 & 0.575 & 2.063 & 10 & 6.64 \\
\hline 1.184 & 7.235 & 3.091 & 26.5 & 0.573 & 2.067 & 10 & 6.67 \\
\hline 1.185 & 7.206 & 3.092 & 26.4 & 0.571 & 2.076 & 10 & 6.67 \\
\hline 1.187 & 7.228 & 3.093 & 26.5 & 0.572 & 2.075 & 10 & 6.68 \\
\hline 1.187 & 7.440 & 3.402 & 30.0 & 0.543 & 2.187 & 10 & 6.95 \\
\hline 1.186 & 7.549 & 3.468 & 31.0 & 0.541 & 2.194 & 10 & 7.01 \\
\hline 1.187 & 7.602 & 3.500 & 31.6 & 0.540 & 2.200 & 1 & 8.68 \\
\hline 1.187 & 7.757 & 3.581 & 33.0 & 0.538 & 2.205 & 10 & 7.12 \\
\hline 1.187 & 7.677 & 3.586 & 32.7 & 0.533 & 2.228 & 10 & 7.12 \\
\hline 1.187 & 7.677 & 3.586 & 32.7 & 0.533 & 2.228 & 10 & 7.12 \\
\hline 1.187 & 7.964 & 3.855 & 36.4 & 0.516 & 2.301 & 10 & 7.37 \\
\hline 1.187 & 7.860 & 3.863 & 36.0 & 0.509 & 2.334 & 10 & 7.37 \\
\hline 1.186 & 7.951 & 3.883 & 36.6 & 0.512 & 2.318 & 10 & 7.39 \\
\hline 1.187 & 8.320 & 3.905 & 38.6 & 0.531 & 2.237 & 10 & 7.43 \\
\hline 1.187 & 8.196 & 3.914 & 38.1 & 0.522 & 2.272 & 10 & 7.43 \\
\hline 1.187 & 8.194 & 3.914 & 38.1 & 0.522 & 2.272 & 10 & 7.43 \\
\hline 1.187 & 8.104 & 3.944 & 37.9 & 0.513 & 2.312 & 10 & 7.45 \\
\hline
\end{tabular}


Polymethylmethacrylate (cont.)

\begin{tabular}{|c|c|c|c|c|c|c|c|}
\hline $\begin{array}{c}\rho_{o} \\
\left(\mathrm{~g} / \mathrm{cm}^{3}\right)\end{array}$ & $\begin{array}{c}u_{s} \\
(\mathrm{~km} / \mathrm{s})\end{array}$ & $\begin{array}{c}u_{p} \\
(\mathrm{~km} / \mathrm{s})\end{array}$ & $\begin{array}{c}P \\
(\mathrm{GPa})\end{array}$ & $V / V_{0}$ & $\begin{array}{c}\rho \\
\left(\mathrm{g} / \mathrm{cm}^{3}\right)\end{array}$ & std & $\begin{array}{c}u_{\text {sstd }} \\
(\mathrm{km} / \mathrm{s})\end{array}$ \\
\hline 1.185 & 8.147 & 4.025 & 38.9 & 0.506 & 2.342 & 1 & 9.21 \\
\hline 1.185 & 8.146 & 4.025 & 38.9 & 0.506 & 2.343 & 1 & 9.21 \\
\hline 1.189 & 8.285 & 4.075 & 40.1 & 0.508 & 2.340 & 10 & 7.57 \\
\hline 1.187 & 8.474 & 4.120 & 41.4 & 0.514 & 2.311 & 1 & 9.33 \\
\hline 1.186 & 8.412 & 4.161 & 41.5 & 0.505 & 2.347 & 10 & 7.65 \\
\hline 1.186 & 8.596 & 4.273 & 43.6 & 0.503 & 2.358 & 10 & 7.76 \\
\hline 1.186 & 8.596 & 4.273 & 43.6 & 0.503 & 2.358 & 10 & 7.76 \\
\hline 1.189 & 8.463 & 4.275 & 43.0 & 0.495 & 2.402 & 10 & 7.75 \\
\hline 1.186 & 8.562 & 4.275 & 43.4 & 0.501 & 2.369 & 10 & 7.76 \\
\hline 1.186 & 8.554 & 4.276 & 43.4 & 0.500 & 2.371 & 10 & 7.76 \\
\hline 1.186 & 8.537 & 4.277 & 43.3 & 0.499 & 2.377 & 10 & 7.76 \\
\hline 1.186 & 8.916 & 4.531 & 47.9 & 0.492 & 2.411 & 1 & 9.76 \\
\hline 1.185 & 8.888 & 4.534 & 47.8 & 0.490 & 2.416 & $\overline{1}$ & 9.76 \\
\hline 1.186 & 9.101 & 4.613 & 49.8 & 0.493 & 2.405 & 10 & 8.08 \\
\hline 1.186 & 9.253 & 4.632 & 50.8 & 0.499 & 2.375 & 1 & 9.89 \\
\hline 1.187 & 9.344 & 4.635 & 51.4 & 0.504 & 2.355 & 10 & 8.11 \\
\hline 1.185 & 9.210 & 4.639 & 50.6 & 0.496 & 2.388 & 1 & 9.89 \\
\hline 1.187 & 9.208 & 4.646 & 50.8 & 0.495 & 2.396 & 10 & 8.11 \\
\hline 1.187 & 9.148 & 4.651 & 50.5 & 0.492 & 2.415 & 10 & 8.11 \\
\hline 1.187 & 9.092 & 4.656 & 50.2 & 0.488 & 2.433 & 10 & 8.11 \\
\hline 1.197 & 9.048 & 4.659 & 50.0 & 0.485 & 2.447 & 10 & 8.11 \\
\hline 1.185 & 9.045 & 4.661 & 50.0 & 0.485 & 2.445 & 1 & 9.89 \\
\hline 1.188 & 9.191 & 4.666 & 50.9 & 0.492 & 2.413 & 10 & 8.13 \\
\hline 1.187 & 9.192 & 4.667 & 50.9 & 0.492 & 2.411 & 10 & 8.13 \\
\hline 1.186 & 9.280 & 4.750 & 52.3 & 0.488 & 2.430 & 1 & 10.00 \\
\hline 1.185 & 9.190 & 4.764 & 51.9 & 0.482 & 2.460 & 1 & 10.00 \\
\hline 1.185 & 9.159 & 4.768 & 51.7 & 0.479 & 2.472 & 1 & 10.00 \\
\hline 1.187 & 9.195 & 4.774 & 52.1 & 0.481 & 2.469 & 10 & 8.22 \\
\hline 1.187 & 9.358 & 4.827 & 53.6 & 0.484 & 2.452 & 10 & 8.28 \\
\hline 1.187 & 9.332 & 4.829 & 53.5 & 0.483 & 2.460 & 10 & 8.28 \\
\hline 1.189 & 9.806 & 5.075 & 59.2 & 0.482 & 2.464 & 1 & 10.35 \\
\hline 1.186 & 9.684 & 5.219 & 59.9 & 0.461 & 2.573 & 1 & 10.46 \\
\hline 1.185 & 9.688 & 5.220 & 59.9 & 0.461 & 2.569 & 1 & 10.46 \\
\hline 1.185 & 9.676 & 5.222 & 59.9 & 0.460 & 2.574 & 1 & 10.46 \\
\hline 1.185 & 9.671 & 5.223 & 59.9 & 0.460 & 2.576 & 1 & 10.46 \\
\hline
\end{tabular}


Polystyrene (Styrolux-Westlake)

\begin{tabular}{|c|c|c|c|c|c|c|c|}
\hline $\begin{array}{c}\rho_{\circ} \\
\left(\mathrm{g} / \mathrm{cm}^{3}\right)\end{array}$ & $\begin{array}{c}u_{s} \\
(\mathrm{~km} / \mathrm{s})\end{array}$ & $\begin{array}{c}u_{p} \\
(\mathrm{~km} / \mathrm{s})\end{array}$ & $\begin{array}{c}P \\
(\mathrm{GPa})\end{array}$ & $V / V_{o}$ & $\begin{array}{c}\rho \\
\left(\mathrm{g} / \mathrm{cm}^{3}\right)\end{array}$ & std & $\begin{array}{c}u_{\text {sstd }} \\
(\mathrm{km} / \mathrm{s})\end{array}$ \\
\hline 1.046 & 1.890 & 0.000 & 0.0 & 1.000 & 1.046 & 40 & $\overline{0.00}$ \\
\hline 1.044 & 3.638 & 0.759 & 2.9 & 0.791 & 1.319 & 1 & 5.96 \\
\hline 1.047 & 3.506 & 0.760 & 2.8 & 0.783 & 1.337 & 1 & 5.96 \\
\hline 1.044 & 3.519 & 0.825 & 3.0 & 0.766 & 1.364 & 1 & 6.01 \\
\hline 1.047 & 3.850 & 0.994 & 4.0 & 0.742 & 1.411 & 1 & 6.16 \\
\hline 1.047 & 3.913 & 1.034 & 4.2 & 0.736 & 1.423 & 1 & 6.20 \\
\hline 1.044 & 4.591 & 1.406 & 6.7 & 0.694 & 1.505 & 1 & 6.54 \\
\hline 1.049 & 4.679 & 1.429 & 7.0 & 0.695 & 1.510 & 1 & 6.57 \\
\hline 1.047 & 4.679 & 1.433 & 7.0 & 0.694 & 1.509 & 1 & 6.57 \\
\hline 1.049 & 5.195 & 1.794 & 9.8 & 0.655 & 1.603 & 1 & 6.91 \\
\hline 1.047 & 5.281 & 1.798 & 9.9 & 0.660 & 1.587 & 1 & 6.92 \\
\hline 1.047 & 6.251 & 2.463 & 16.1 & 0.606 & 1.728 & 1 & 7.56 \\
\hline 1.046 & 6.233 & 2.507 & 16.3 & 0.599 & 1.750 & 1 & 7.60 \\
\hline 1.047 & 6.560 & 2.671 & 18.3 & 0.593 & 1.766 & 1 & 7.77 \\
\hline 1.047 & 6.742 & 2.799 & 19.8 & 0.585 & 1.790 & 1 & 7.90 \\
\hline 1.047 & 6.994 & 3.217 & 23.6 & 0.540 & 1.939 & 1 & 8.28 \\
\hline 1.044 & 7.019 & 3.418 & 25.0 & 0.513 & 2.035 & 1 & 8.46 \\
\hline 1.047 & 7.091 & 3.466 & 25.7 & 0.511 & 2.048 & 1 & 8.51 \\
\hline 1.044 & 7.257 & 3.707 & 28.1 & 0.489 & 2.134 & 1 & 8.73 \\
\hline 1.047 & 7.528 & 3.968 & 31.3 & 0.473 & 2.214 & 1 & 8.98 \\
\hline 1.047 & 7.851 & 4.189 & 34.4 & 0.466 & 2.244 & 1 & 9.21 \\
\hline 1.047 & 7.873 & 4.215 & 34.7 & 0.465 & 2.254 & 1 & 9.24 \\
\hline 1.047 & 8.145 & 4.288 & 36.6 & 0.474 & 2.211 & 1 & 9.33 \\
\hline 1.044 & 8.352 & 4.468 & 39.0 & 0.465 & 2.245 & 1 & 9.51 \\
\hline 1.045 & 8.818 & 4.713 & 43.4 & 0.465 & 2.245 & 1 & 9.77 \\
\hline 1.045 & 8.784 & 4.736 & 43.5 & 0.461 & 2.267 & 1 & 9.79 \\
\hline 1.047 & 8.888 & 4.869 & 45.3 & 0.452 & 2.315 & 1 & 9.92 \\
\hline 1.049 & 8.980 & 4.900 & 46.2 & 0.454 & 2.309 & 1 & 9.96 \\
\hline 1.047 & 8.879 & 4.912 & 45.7 & 0.447 & 2.343 & 1 & 9.95 \\
\hline 1.047 & 8.848 & 4.953 & 45.9 & 0.440 & 2.378 & 1 & 9.98 \\
\hline 1.047 & 8.949 & 4.973 & 46.6 & 0.444 & 2.356 & 1 & 10.01 \\
\hline 1.045 & 9.570 & 5.336 & 53.4 & 0.442 & 2.362 & 1 & 10.40 \\
\hline 1.045 & 9.556 & 5.367 & 53.6 & 0.438 & 2.384 & 1 & 10.43 \\
\hline
\end{tabular}

Cellulose Acetate (Tenite 1-Eastman)

\begin{tabular}{|c|c|c|c|c|c|c|c|}
\hline $\begin{array}{c}\rho_{o} \\
\left(\mathrm{~g} / \mathrm{cm}^{3}\right)\end{array}$ & $\begin{array}{c}u_{s} \\
(\mathrm{~km} / \mathrm{s})\end{array}$ & $\begin{array}{c}u_{p} \\
(\mathrm{~km} / \mathrm{s})\end{array}$ & $\begin{array}{c}P \\
(\mathrm{GPa})\end{array}$ & $V / V_{o}$ & $\begin{array}{c}\rho \\
\left(\mathrm{g} / \mathrm{cm}^{3}\right)\end{array}$ & std & $\begin{array}{c}u_{s s t d} \\
(\mathrm{~km} / \mathrm{s})\end{array}$ \\
\hline 1.255 & 1.592 & 0.000 & 0.0 & 1.000 & 1.255 & 40 & 0.00 \\
\hline 1.260 & 3.339 & 0.672 & 2.8 & 1.799 & 1.577 & 1 & 5.90 \\
\hline 1.260 & 3.799 & 0.995 & 4.8 & 1.738 & 1.707 & 1 & 6.19 \\
\hline 1.260 & 3.799 & 0.995 & 4.8 & 0.738 & 1.707 & 1 & 6.19 \\
\hline 1.260 & 4.458 & 1.374 & 7.7 & 0.692 & 1.822 & 1 & 6.56 \\
\hline 1.260 & 5.050 & 1.781 & 11.3 & 0.647 & 1.947 & 1 & 6.96 \\
\hline 1.260 & 6.013 & 2.406 & 18.2 & 0.600 & 2.100 & 1 & 6.59 \\
\hline 1.260 & 6.322 & 2.593 & 20.7 & 0.590 & 5.136 & 1 & 7.79 \\
\hline 1.260 & 6.484 & 2.857 & 23.3 & 0.559 & 2.252 & 1 & 8.04 \\
\hline 1.260 & 6.767 & 3.141 & 26.8 & 0.536 & 2.351 & 1 & 8.32 \\
\hline 1.260 & 6.918 & 3.306 & 28.8 & 0.522 & 2.413 & 1 & 8.49 \\
\hline 1.260 & 7.198 & 3.531 & 32.0 & 0.509 & 2.473 & 1 & 8.72 \\
\hline 1.260 & 7.730 & 3.855 & 37.5 & 0.501 & 2.513 & 1 & 9.07 \\
\hline 1.260 & 8.833 & 4.638 & 51.6 & 0.475 & 2.653 & 1 & 9.91 \\
\hline 1.260 & 9.016 & 4.785 & 54.4 & 0.469 & 2.685 & 1 & 10.06 \\
\hline 1.260 & 9.425 & 5.117 & 60.8 & 0.457 & 2.757 & 1 & 10.41 \\
\hline
\end{tabular}


Epoxy (Epon 828-Shell)

\begin{tabular}{|c|c|c|c|c|c|c|c|}
\hline $\begin{array}{c}\rho_{0} \\
\left(\mathrm{~g} / \mathrm{cm}^{3}\right)\end{array}$ & $\begin{array}{c}u_{s} \\
(\mathrm{~km} / \mathrm{s})\end{array}$ & $\begin{array}{c}u_{p} \\
(\mathrm{~km} / \mathrm{s})\end{array}$ & $\begin{array}{c}P \\
(\mathrm{GPa})\end{array}$ & $V / V_{o}$ & $\begin{array}{c}\rho \\
\left(\mathrm{g} / \mathrm{cm}^{3}\right)\end{array}$ & std & $\begin{array}{c}u_{\text {sstd }} \\
(\mathrm{km} / \mathrm{s})\end{array}$ \\
\hline 1.192 & 2.256 & 0.000 & 0.0 & 1.000 & 1.192 & 40 & 0.00 \\
\hline 1.184 & 3.265 & 0.370 & 1.4 & 0.887 & 1.335 & 10 & 4.24 \\
\hline 1.184 & 3.355 & 0.410 & 1.6 & 0.878 & 1.349 & 1 & 5.67 \\
\hline 1.184 & 3.421 & 0.494 & 2.0 & 0.856 & 1.384 & $\overline{1}$ & 5.75 \\
\hline 1.184 & 3.805 & 0.639 & 2.9 & 0.832 & 1.423 & 10 & 4.47 \\
\hline 1.184 & 3.709 & 0.699 & 3.1 & 0.812 & 1.459 & 1 & 5.93 \\
\hline 1.184 & 3.798 & 0.716 & 3.2 & 0.811 & 1.459 & 1 & 5.94 \\
\hline 1.184 & 3.703 & 0.742 & 3.3 & 0.799 & 1.481 & 1 & 5.96 \\
\hline 1.184 & 3.717 & 0.780 & 3.4 & 0.790 & 1.498 & 1 & 6.00 \\
\hline 1.184 & 4.312 & 0.924 & 4.7 & 0.786 & 1.507 & 10 & 4.72 \\
\hline 1.184 & 4.168 & 0.926 & 4.6 & 0.778 & 1.522 & 1 & 6.14 \\
\hline 1.184 & 4.225 & 0.960 & 4.8 & 0.773 & 1.532 & 10 & 4.75 \\
\hline 1.184 & 4.190 & 0.961 & 4.8 & 0.771 & 1.537 & 10 & 4.75 \\
\hline 1.184 & 4.086 & 0.989 & 4.8 & 0.758 & 1.562 & 1 & 6.19 \\
\hline 1.184 & 4.129 & 0.998 & 4.9 & 0.758 & 1.556 & 1 & 6.20 \\
\hline 1.184 & 4.480 & 1.151 & 6.1 & 0.743 & 1.593 & 10 & 4.92 \\
\hline 1.184 & 4.575 & 1.194 & 6.5 & 0.739 & 1.602 & 10 & 4.96 \\
\hline 1.184 & 4.558 & 1.195 & 6.4 & 0.738 & 1.605 & 10 & 4.96 \\
\hline 1.184 & 4.742 & 1.345 & 7.6 & 0.716 & 1.653 & 1 & 6.53 \\
\hline 1.184 & 4.718 & 1.340 & 7.5 & 0.714 & 1.652 & 1 & 6.53 \\
\hline 1.184 & 4.742 & 1.366 & 7.7 & 0.712 & 1.663 & 1 & 6.55 \\
\hline 1.184 & 4.681 & 1.425 & 7.9 & 0.696 & 1.702 & 1 & 6.60 \\
\hline 1.184 & 5.305 & 1.701 & 7.9 & 0.696 & 1.702 & 1 & 6.60 \\
\hline 1.184 & 5.305 & 1.701 & 10.7 & 0.679 & 1.743 & 10 & 5.41 \\
\hline 1.184 & 5.370 & 1.717 & 10.9 & 0.680 & 1.740 & 10 & 5.42 \\
\hline 1.184 & 5.365 & 1.735 & 11.0 & 0.677 & 1.750 & 1 & 6.91 \\
\hline 1.184 & 5.327 & 1.746 & 11.0 & 0.672 & 1.762 & 1 & 6.92 \\
\hline 1.184 & 5.311 & 1.787 & 11.2 & 0.664 & 1.778 & 1 & 6.95 \\
\hline 1.184 & 5.349 & 1.798 & 11.4 & 0.664 & 1.784 & 1 & 6.97 \\
\hline 1.184 & 5.330 & 1.811 & 11.4 & 0.660 & 1.793 & 10 & 5.50 \\
\hline 1.184 & 5.626 & 1.893 & 12.6 & 0.663 & 1.785 & 10 & 5.58 \\
\hline 1.184 & 6.343 & 2.358 & 17.7 & 0.628 & 1.885 & 1 & 7.54 \\
\hline 1.184 & 6.281 & 2.393 & 17.8 & 0.619 & 1.913 & 1 & 7.57 \\
\hline 1.184 & 6.380 & 2.400 & 18.1 & 0.624 & 1.898 & 1 & 7.58 \\
\hline 1.184 & 6.628 & 2.410 & 18.9 & 0.636 & 1.861 & 1 & 7.61 \\
\hline 1.184 & 6.433 & 2.520 & 19.2 & 0.608 & 1.946 & 10 & 6.15 \\
\hline 1.180 & 6.509 & 2.553 & 19.6 & 0.608 & 1.941 & 1 & 7.73 \\
\hline 1.180 & 6.603 & 2.575 & 20.1 & 0.610 & 1.941 & 1 & 7.76 \\
\hline 1.180 & 6.641 & 2.610 & 20.5 & 0.607 & 1.944 & 1 & 7.79 \\
\hline 1.180 & 6.881 & 2.753 & 22.4 & 0.600 & 1.967 & 1 & 7.74 \\
\hline 1.184 & 6.880 & 2.761 & 22.5 & 0.599 & 1.978 & 1 & 7.95 \\
\hline 1.184 & 7.166 & 2.844 & 24.1 & 0.603 & 1.963 & 10 & 6.46 \\
\hline 1.180 & 7.201 & 3.023 & 25.7 & 0.580 & 2.034 & 1 & 8.21 \\
\hline 1.184 & 7.214 & 3.177 & 27.1 & 0.560 & 2.116 & 10 & 6.75 \\
\hline 1.184 & 7.343 & 3.190 & 27.7 & 0.566 & 2.094 & 1 & 8.38 \\
\hline 1.180 & 7.471 & 3.361 & 29.6 & 0.560 & 2.145 & 1 & 8.54 \\
\hline 1.184 & 7.612 & 3.558 & 32.1 & 0.533 & 2.223 & 1 & 8.74 \\
\hline 1.184 & 7.652 & 3.576 & 32.4 & 0.533 & 2.223 & 1 & 8.76 \\
\hline 1.180 & 7.697 & 3.590 & 32.6 & 0.534 & 2.212 & 1 & 8.77 \\
\hline 1.184 & 7.624 & 3.608 & 32.6 & 0.527 & 2.247 & 10 & 7.13 \\
\hline 1.184 & 7.954 & 3.784 & 35.6 & 0.524 & 2.258 & 10 & 7.30 \\
\hline 1.184 & 8.388 & 3.891 & 38.6 & 0.536 & 2.208 & 10 & 7.42 \\
\hline 1.184 & 8.244 & 3.949 & 38.5 & 0.521 & 2.273 & 1 & 9.16 \\
\hline 1.184 & 8.063 & 3.954 & 37.8 & 0.510 & 2.324 & 10 & 7.45 \\
\hline 1.180 & 8.286 & 3.968 & 38.8 & 0.521 & 2.264 & 1 & 9.17 \\
\hline 1.181 & 8.483 & 4.143 & 41.5 & 0.512 & 2.309 & 10 & 7.64 \\
\hline 1.180 & 8.627 & 4.220 & 43.0 & 0.511 & 2.310 & 1 & 9.44 \\
\hline 1.180 & 8.592 & 4.254 & 43.1 & 0.505 & 2.337 & 1 & 9.46 \\
\hline 1.184 & 8.896 & 4.495 & 47.3 & 0.495 & 2.393 & 1 & 9.72 \\
\hline 1.180 & 9.159 & 4.556 & 49.2 & 0.503 & 2.348 & 1 & 9.80 \\
\hline 1.184 & 9.158 & 4.678 & 50.7 & 0.489 & 2.421 & 10 & 8.14 \\
\hline 1.180 & 9.201 & 4.703 & 51.1 & 0.489 & 2.414 & 1 & 9.94 \\
\hline 1.180 & 9.369 & 4.823 & 53.3 & 0.485 & 2.432 & 1 & 10.06 \\
\hline 1.184 & 9.739 & 5.098 & 58.8 & 0.477 & 2.485 & 1 & 10.36 \\
\hline 1.184 & 9.756 & 5.128 & 59.2 & 0.474 & 2.496 & 1 & 10.39 \\
\hline 1.180 & 9.989 & 5.131 & 60.5 & 0.486 & 2.426 & 1 & 10.42 \\
\hline 1.180 & 9.757 & 5.189 & 59.7 & 0.468 & 2.521 & 1 & 10.44 \\
\hline
\end{tabular}


Phenolic (Durite HR 300-Borden)

\begin{tabular}{rrrrrrrr}
\hline \hline $\begin{array}{c}\rho_{o} \\
\left(\mathrm{~g} / \mathrm{cm}^{3}\right)\end{array}$ & $\begin{array}{c}u_{s} \\
(\mathrm{~km} / \mathrm{s})\end{array}$ & $\begin{array}{c}u_{p} \\
(\mathrm{~km} / \mathrm{s})\end{array}$ & $\begin{array}{c}P \\
(\mathrm{GPa})\end{array}$ & $V / V_{o}$ & $\begin{array}{c}\rho \\
\left(\mathrm{g} / \mathrm{cm}^{3}\right)\end{array}$ & std & $\begin{array}{c}u_{s s t d} \\
(\mathrm{~km} / \mathrm{s})\end{array}$ \\
\hline 1.388 & 2.440 & 0.000 & 0.0 & 1.000 & 1.388 & 40 & 0.00 \\
1.388 & 3.973 & 0.689 & 3.8 & 0.827 & 1.679 & 1 & 5.95 \\
1.379 & 4.032 & 0.698 & 3.9 & 0.827 & 1.668 & 1 & 5.96 \\
1.388 & 3.895 & 0.760 & 4.1 & 0.805 & 1.724 & 1 & 6.01 \\
1.389 & 4.188 & 0.916 & 5.3 & 0.781 & 1.778 & 1 & 6.16 \\
1.388 & 4.299 & 0.950 & 5.7 & 0.779 & 1.782 & 1 & 6.20 \\
1.388 & 4.923 & 1.320 & 9.0 & 0.732 & 1.896 & 1 & 6.57 \\
1.388 & 5.339 & 1.664 & 12.3 & 0.688 & 2.016 & 1 & 6.91 \\
1.388 & 5.424 & 1.720 & 12.9 & 0.683 & 2.032 & 1 & 6.97 \\
1.388 & 6.252 & 2.321 & 20.1 & 0.629 & 2.207 & 1 & 7.59 \\
1.388 & 6.109 & 2.345 & 19.9 & 0.616 & 2.253 & 1 & 7.60 \\
1.388 & 6.406 & 2.460 & 21.9 & 0.616 & 2.254 & 1 & 7.73 \\
1.374 & 6.518 & 2.522 & 22.6 & 0.613 & 2.241 & 1 & 7.79 \\
1.369 & 6.835 & 3.035 & 28.4 & 0.556 & 2.462 & 1 & 8.29 \\
1.387 & 7.160 & 3.241 & 32.2 & 0.547 & 2.534 & 1 & 8.53 \\
1.370 & 7.261 & 3.429 & 34.1 & 0.528 & 2.596 & 1 & 8.70 \\
1.388 & 7.713 & 3.645 & 39.0 & 0.527 & 2.631 & 1 & 8.97 \\
1.388 & 7.979 & 3.876 & 42.9 & 0.514 & 2.699 & 1 & 8.21 \\
1.388 & 8.555 & 4.199 & 49.9 & 0.509 & 2.726 & 1 & 9.58 \\
1.388 & 8.880 & 4.378 & 54.0 & 0.507 & 2.738 & 1 & 9.79 \\
1.388 & 9.162 & 4.524 & 57.5 & 0.506 & 2.742 & 1 & 9.96 \\
1.388 & 9.607 & 4.861 & 64.8 & 0.494 & 2.810 & 1 & 10.33 \\
1.388 & 9.740 & 4.922 & 66.5 & 0.495 & 2.806 & 1 & 10.40 \\
1.388 & 9.656 & 4.960 & 66.5 & 0.487 & 2.851 & 1 & 10.43 \\
1.388 & 9.656 & 4.960 & 66.5 & 0.487 & 2.851 & 1 & 10.43 \\
\hline \hline
\end{tabular}

Phenoxy (PRDA8060-Union Carbide)

\begin{tabular}{cccccrrr}
\hline $\begin{array}{c}\rho_{o} \\
\left(\mathrm{~g} / \mathrm{cm}^{3}\right)\end{array}$ & $\begin{array}{c}u_{s} \\
(\mathrm{~km} / \mathrm{s})\end{array}$ & $\begin{array}{c}u_{p} \\
(\mathrm{~km} / \mathrm{s})\end{array}$ & $\begin{array}{c}P \\
(\mathrm{GPa})\end{array}$ & $V / V_{o}$ & $\begin{array}{c}\rho \\
\left(\mathrm{g} / \mathrm{cm}^{3}\right)\end{array}$ & std & $\begin{array}{c}u_{\text {sstd }} \\
(\mathrm{km} / \mathrm{s})\end{array}$ \\
\hline 1.178 & 2.173 & 0.000 & 0.0 & 1.000 & 1.178 & 40 & 0.00 \\
1.181 & 3.699 & 0.666 & 2.9 & 0.820 & 1.440 & 1 & 5.90 \\
1.181 & 4.088 & 0.993 & 4.8 & 0.757 & 1.560 & 1 & 6.19 \\
1.181 & 4.769 & 1.374 & 7.7 & 0.712 & 1.659 & 1 & 6.56 \\
1.181 & 5.385 & 1.781 & 11.3 & 0.669 & 1.765 & 1 & 6.96 \\
1.181 & 6.344 & 2.413 & 18.1 & 0.620 & 1.906 & 1 & 7.59 \\
1.181 & 6.642 & 2.603 & 20.4 & 0.608 & 1.942 & 1 & 7.79 \\
1.181 & 6.870 & 2.862 & 23.2 & 0.583 & 2.024 & 1 & 8.04 \\
1.181 & 7.133 & 3.150 & 26.5 & 0.558 & 2.115 & 1 & 8.32 \\
1.181 & 7.199 & 3.327 & 28.3 & 0.538 & 2.196 & 1 & 8.49 \\
1.181 & 7.415 & 3.563 & 31.2 & 0.519 & 2.274 & 1 & 8.72 \\
1.181 & 8.920 & 4.631 & 48.8 & 0.481 & 2.456 & 1 & 9.84 \\
1.181 & 9.236 & 4.837 & 52.8 & 0.476 & 2.479 & 1 & 10.06 \\
1.181 & 9.684 & 5.169 & 59.1 & 0.466 & 2.533 & 1 & 10.41 \\
\hline \hline
\end{tabular}

Polycarbonate (Lexan)

\begin{tabular}{|c|c|c|c|c|c|c|c|}
\hline $\begin{array}{c}\rho_{o} \\
\left(\mathrm{~g} / \mathrm{cm}^{3}\right)\end{array}$ & $\begin{array}{c}u_{s} \\
(\mathrm{~km} / \mathrm{s})\end{array}$ & $\begin{array}{c}u_{p} \\
(\mathrm{~km} / \mathrm{s})\end{array}$ & $\begin{array}{c}P \\
(\mathrm{GPa})\end{array}$ & $V / V_{o}$ & $\begin{array}{c}\rho \\
\left(\mathrm{g} / \mathrm{cm}^{3}\right)\end{array}$ & std & $\begin{array}{c}u_{s s t d} \\
(\mathrm{~km} / \mathrm{s})\end{array}$ \\
\hline 1.194 & 1.928 & 0.000 & 0.0 & 1.000 & 1.194 & 40 & 0.00 \\
\hline 1.196 & 3.566 & 0.724 & 3.1 & 0.797 & 1.501 & 1 & 5.94 \\
\hline 1.196 & 3.891 & 0.938 & 4.4 & 0.759 & 1.576 & 1 & 6.14 \\
\hline 1.196 & 4.517 & 1.357 & 7.3 & 0.700 & 1.710 & 1 & 6.53 \\
\hline 1.196 & 4.599 & 1.396 & 7.7 & 0.696 & 1.717 & 1 & 6.57 \\
\hline 1.196 & 5.194 & 1.750 & 10.9 & 0.663 & 1.804 & 1 & 6.92 \\
\hline 1.196 & 5.115 & 1.750 & 10.7 & 0.658 & 1.818 & 1 & 6.91 \\
\hline 1.196 & 5.112 & 1.781 & 10.9 & 0.652 & 1.835 & 1 & 6.94 \\
\hline 1.196 & 6.069 & 2.379 & 17.3 & 0.608 & 1.967 & 1 & 7.54 \\
\hline 1.196 & 6.515 & 2.793 & 21.8 & 0.571 & 2.093 & 1 & 7.95 \\
\hline 1.196 & 6.671 & 3.106 & 24.8 & 0.534 & 2.238 & 1 & 8.24 \\
\hline 1.196 & 6.750 & 3.251 & 26.2 & 0.518 & 2.307 & 1 & 8.38 \\
\hline 1.196 & 6.766 & 3.336 & 27.0 & 0.507 & 2.359 & 1 & 8.46 \\
\hline 1.196 & 6.815 & 3.388 & 27.6 & 0.503 & 2.378 & 1 & 8.51 \\
\hline 1.196 & 7.023 & 3.615 & 30.4 & 0.485 & 2.465 & 1 & 8.73 \\
\hline 1.196 & 7.072 & 3.640 & 30.8 & 0.485 & 2.464 & 1 & 8.76 \\
\hline 1.196 & 7.705 & 4.009 & 36.9 & 0.480 & 2.493 & 1 & 9.16 \\
\hline 1.196 & 8.187 & 4.208 & 41.2 & 0.486 & 2.461 & 1 & 9.39 \\
\hline 1.196 & 8.592 & 4.524 & 46.5 & 0.473 & 2.526 & 1 & 9.72 \\
\hline 1.196 & 9.305 & 5.179 & 57.6 & 0.443 & 2.697 & 1 & 10.39 \\
\hline
\end{tabular}


Polycarbonate (Merlon)

\begin{tabular}{|c|c|c|c|c|c|c|c|}
\hline $\begin{array}{c}\rho_{o} \\
\left(\mathrm{~g} / \mathrm{cm}^{3}\right) \\
\end{array}$ & $\begin{array}{c}u_{s} \\
(\mathrm{~km} / \mathrm{s})\end{array}$ & $\begin{array}{c}u_{p} \\
(\mathrm{~km} / \mathrm{s})\end{array}$ & $\begin{array}{c}P \\
(\mathrm{GPa})\end{array}$ & $V / V_{o}$ & $\begin{array}{c}\rho \\
\left(\mathrm{g} / \mathrm{cm}^{3}\right)\end{array}$ & std & $\begin{array}{c}u_{s s t d} \\
(\mathrm{~km} / \mathrm{s})\end{array}$ \\
\hline 1.191 & 1.924 & 0.000 & 0.0 & 1.000 & 1.191 & 40 & 0.00 \\
\hline 1.191 & 2.949 & 0.420 & 1.5 & 0.858 & 1.389 & 1 & 5.67 \\
\hline 1.191 & 3.189 & 0.501 & 1.9 & 0.843 & 1.413 & 1 & 5.75 \\
\hline 1.191 & 3.380 & 0.647 & 2.6 & 0.809 & 1.473 & 10 & 4.47 \\
\hline 1.191 & 3.506 & 0.727 & 3.0 & 0.793 & 1.503 & 1 & 5.94 \\
\hline 1.191 & 3.465 & 0.752 & 3.1 & 0.783 & 1.521 & 1 & 5.96 \\
\hline 1.191 & 3.987 & 0.931 & 4.4 & 0.766 & 1.554 & 10 & 4.72 \\
\hline 1.191 & 3.861 & 0.940 & 4.3 & 0.756 & 1.574 & 1 & 6.14 \\
\hline 1.191 & 3.815 & 0.976 & 4.4 & 0.744 & 1.600 & 1 & 6.17 \\
\hline 1.191 & 3.880 & 0.999 & 4.6 & 0.742 & 1.604 & 1 & 6.19 \\
\hline 1.191 & 4.191 & 1.159 & 5.8 & 0.723 & 1.646 & 10 & 4.92 \\
\hline 1.191 & 4.321 & 1.279 & 6.6 & 0.704 & 1.692 & 1 & 6.45 \\
\hline 1.191 & 4.522 & 1.358 & 7.3 & 0.700 & 1.702 & 1 & 6.53 \\
\hline 1.191 & 4.378 & 1.359 & 7.1 & 0.690 & 1.727 & 1 & 6.52 \\
\hline 1.191 & 4.529 & 1.378 & 7.4 & 0.596 & 1.712 & 1 & 6.55 \\
\hline 1.191 & 4.541 & 1.429 & 7.7 & 0.685 & 1.738 & 1 & 6.59 \\
\hline 1.191 & 4.979 & 1.684 & 10.0 & 0.662 & 1.800 & 1 & 6.84 \\
\hline 1.191 & 5.113 & 1.752 & 10.7 & 0.657 & 1.812 & 1 & 6.91 \\
\hline 1.191 & 5.133 & 1.760 & 10.8 & 0.657 & 1.812 & 1 & 6.92 \\
\hline 1.191 & 5.123 & 1.818 & 11.1 & 0.645 & 1.846 & 10 & 5.50 \\
\hline 1.191 & 6.040 & 2.384 & 17.1 & 0.605 & 1.968 & 11 & 7.54 \\
\hline 1.191 & 6.092 & 2.408 & 17.5 & 0.605 & 1.969 & 11 & 7.57 \\
\hline 1.191 & 6.305 & 2.438 & 18.3 & 0.613 & 1.942 & 1 & 7.61 \\
\hline 1.191 & 6.215 & 2.530 & 18.7 & 0.593 & 2.009 & 10 & 6.15 \\
\hline 1.191 & 6.512 & 2.796 & 21.7 & 0.571 & 2.087 & 1 & 7.95 \\
\hline 1.191 & 6.666 & 2.872 & 22.8 & 0.569 & 2.092 & 10 & 6.46 \\
\hline 1.191 & 6.652 & 3.186 & 25.2 & 0.521 & 2.286 & 1 & 8.31 \\
\hline 1.191 & 6.649 & 3.212 & 25.4 & 0.517 & 2.304 & 10 & 6.75 \\
\hline 1.191 & 6.811 & 3.247 & 26.3 & 0.523 & 2.276 & 1 & 8.38 \\
\hline 1.191 & 7.082 & 3.642 & 30.7 & 0.486 & 2.452 & 1 & 8.76 \\
\hline 1.191 & 6.989 & 3.651 & 30.4 & 0.478 & 2.494 & 10 & 7.13 \\
\hline 1.191 & 7.527 & 3.992 & 35.8 & 0.470 & 2.536 & 10 & 7.45 \\
\hline 1.191 & 7.673 & 4.017 & 36.7 & 0.476 & 2.500 & 1 & 9.16 \\
\hline 1.192 & 7.769 & 4.149 & 38.4 & 0.466 & 2.558 & 10 & 7.60 \\
\hline 1.192 & 7.788 & 4.192 & 38.9 & 0.462 & 2.582 & 10 & 7.64 \\
\hline 1.192 & 8.152 & 4.218 & 40.9 & 0.483 & 2.468 & 1 & 9.39 \\
\hline 1.192 & 8.550 & 4.535 & 46.2 & 0.470 & 2.536 & 1 & 9.72 \\
\hline 1.192 & 8.562 & 4.671 & 47.6 & 0.454 & 2.621 & 10 & 8.13 \\
\hline 1.192 & 9.108 & 5.213 & 56.6 & 0.428 & 2.785 & 1 & 10.39 \\
\hline
\end{tabular}

Polyester (Clear Cast)

\begin{tabular}{|c|c|c|c|c|c|c|c|}
\hline $\begin{array}{c}\rho_{o} \\
\left(\mathrm{~g} / \mathrm{cm}^{3}\right)\end{array}$ & $\begin{array}{c}u_{s} \\
(\mathrm{~km} / \mathrm{s})\end{array}$ & $\begin{array}{c}u_{p} \\
(\mathrm{~km} / \mathrm{s})\end{array}$ & $\begin{array}{c}P \\
(\mathrm{GPa})\end{array}$ & $V / V_{o}$ & $\begin{array}{c}\rho \\
\left(\mathrm{g} / \mathrm{cm}^{3}\right)\end{array}$ & std & $\begin{array}{c}u_{\text {sstd }} \\
(\mathrm{km} / \mathrm{s})\end{array}$ \\
\hline 1.210 & 2.070 & 0.000 & 0.0 & 1.000 & 1.210 & 40 & 0.00 \\
\hline 1.210 & 3.610 & 0.701 & 3.1 & 0.806 & 1.502 & 1 & 5.93 \\
\hline 1.210 & 3.928 & 0.896 & 4.3 & 0.772 & 1.567 & 1 & 6.11 \\
\hline 1.210 & 3.984 & 0.999 & 4.8 & 0.749 & 1.615 & $\overline{1}$ & 6.20 \\
\hline 1.210 & 4.611 & 1.359 & 7.6 & 0.705 & 1.716 & 1 & 6.54 \\
\hline 1.210 & 4.638 & 1.386 & 7.8 & 0.701 & 1.726 & 1 & 6.57 \\
\hline 1.210 & 5.182 & 1.737 & 10.9 & 0.665 & 1.820 & 1 & 6.91 \\
\hline 1.210 & 5.200 & 1.768 & 11.1 & 0.660 & 1.833 & 1 & 6.94 \\
\hline 1.210 & 5.234 & 1.798 & 11.4 & 0.656 & 1.843 & 1 & 6.97 \\
\hline 1.210 & 6.159 & 2.417 & 18.0 & 0.608 & 1.991 & 1 & 7.59 \\
\hline 1.210 & 6.186 & 2.425 & 18.1 & 0.608 & 1.990 & 1 & 7.60 \\
\hline 1.210 & 6.443 & 2.617 & 20.4 & 0.594 & 2.038 & 1 & 7.79 \\
\hline 1.210 & 6.735 & 3.147 & 25.6 & 0.533 & 2.271 & 1 & 8.29 \\
\hline 1.210 & 6.920 & 3.391 & 28.4 & 0.510 & 2.373 & 1 & 8.53 \\
\hline 1.210 & 7.148 & 3.558 & 30.8 & 0.502 & 2.409 & 1 & 8.70 \\
\hline 1.210 & 7.497 & 3.810 & 34.6 & 0.492 & 2.460 & 1 & 8.97 \\
\hline 1.210 & 7.612 & 3.822 & 35.2 & 0.498 & 2.430 & 1 & 8.99 \\
\hline 1.210 & 7.800 & 4.047 & 38.2 & 0.481 & 2.515 & 1 & 9.21 \\
\hline 1.210 & 8.439 & 4.376 & 44.7 & 0.481 & 2.513 & 1 & 9.58 \\
\hline 1.210 & 8.636 & 4.580 & 47.9 & 0.470 & 2.577 & $\overline{1}$ & 9.79 \\
\hline 1.210 & 8.678 & 4.685 & 49.2 & 0.460 & 2.630 & 1 & 9.89 \\
\hline 1.210 & 8.925 & 4.732 & $\mathbf{5 1 . 1}$ & 0.470 & 2.576 & 1 & 9.96 \\
\hline 1.210 & 9.296 & 5.095 & 57.3 & 0.452 & 2.677 & 1 & 10.33 \\
\hline 1.210 & 9.311 & 5.099 & 57.4 & 0.452 & 2.675 & 1 & 10.33 \\
\hline
\end{tabular}


Polyimide (Melden P1-Dixon)

\begin{tabular}{rrrrrrrr}
\hline $\begin{array}{c}\rho_{o} \\
\left(\mathrm{~g} / \mathrm{cm}^{3}\right)\end{array}$ & $\begin{array}{c}u_{s} \\
(\mathrm{~km} / \mathrm{s})\end{array}$ & $\begin{array}{c}u_{p} \\
(\mathrm{~km} / \mathrm{s})\end{array}$ & $\begin{array}{c}P \\
(\mathrm{GPa})\end{array}$ & $V / V_{o}$ & $\begin{array}{c}\rho \\
\left(\mathrm{g} / \mathrm{cm}^{3}\right)\end{array}$ & std & $\begin{array}{c}u_{\text {sstd }} \\
(\mathrm{km} / \mathrm{s})\end{array}$ \\
\hline 1.414 & 2.332 & 0.000 & 0.0 & 1.000 & 1.414 & 40 & 0.00 \\
1.414 & 3.681 & 0.644 & 3.4 & 0.825 & 1.714 & 1 & 5.90 \\
1.414 & 3.670 & 0.706 & 3.7 & 0.808 & 1.751 & 1 & 5.96 \\
1.414 & 3.999 & 0.935 & 5.3 & 0.766 & 1.846 & 1 & 6.17 \\
1.414 & 4.594 & 1.330 & 8.6 & 0.710 & 1.990 & 1 & 6.56 \\
1.414 & 4.685 & 1.334 & 8.8 & 0.715 & 1.977 & 1 & 6.57 \\
1.414 & 5.210 & 1.675 & 12.3 & 0.678 & 2.084 & 1 & 6.90 \\
1.414 & 5.153 & 1.714 & 12.5 & 0.667 & 2.119 & 1 & 6.95 \\
1.414 & 5.980 & 2.037 & 19.5 & 0.614 & 2.302 & 1 & 7.56 \\
1.414 & 5.931 & 2.312 & 19.4 & 0.610 & 2.318 & 1 & 7.56 \\
1.414 & 6.135 & 2.516 & 21.8 & 0.590 & 2.397 & 1 & 7.77 \\
1.414 & 6.060 & 2.562 & 22.0 & 0.577 & 2.450 & 1 & 7.80 \\
1.414 & 6.096 & 2.636 & 22.7 & 0.568 & 2.491 & 1 & 7.87 \\
1.414 & 6.200 & 2.648 & 23.2 & 0.573 & 2.458 & 1 & 7.90 \\
1.414 & 6.374 & 3.052 & 27.5 & 0.521 & 2.713 & 1 & 8.28 \\
1.414 & 6.311 & 3.128 & 27.9 & 0.504 & 2.804 & 1 & 8.34 \\
1.414 & 6.486 & 3.286 & 30.1 & 0.493 & 2.866 & 1 & 8.51 \\
1.414 & 6.514 & 3.341 & 30.8 & 0.487 & 2.903 & 1 & 8.56 \\
1.414 & 6.762 & 3.530 & 33.7 & 0.478 & 2.958 & 1 & 8.76 \\
1.414 & 7.165 & 3.794 & 38.4 & 0.470 & 3.006 & 1 & 9.05 \\
1.414 & 7.742 & 4.178 & 45.7 & 0.460 & 3.072 & 1 & 9.48 \\
1.414 & 8.299 & 4.503 & 52.8 & 0.457 & 3.091 & 1 & 9.84 \\
1.414 & 8.364 & 4.552 & 53.8 & 0.456 & 3.103 & 1 & 9.90 \\
1.414 & 8.517 & 4.573 & 55.1 & 0.463 & 3.054 & 1 & 9.94 \\
1.414 & 8.578 & 4.626 & 56.1 & 0.461 & 3.070 & 1 & 9.99 \\
1.414 & 8.542 & 4.632 & 55.9 & 0.458 & 3.089 & 1 & 9.99 \\
\hline \hline
\end{tabular}

Polyphenylquinoxyline (NOL)

\begin{tabular}{|c|c|c|c|c|c|c|c|}
\hline $\begin{array}{c}\rho_{o} \\
\left(\mathrm{~g} / \mathrm{cm}^{3}\right) \\
\end{array}$ & $\begin{array}{c}u_{s} \\
(\mathrm{~km} / \mathrm{s})\end{array}$ & $\begin{array}{c}u_{p} \\
(\mathrm{~km} / \mathrm{s})\end{array}$ & $\begin{array}{c}P \\
(\mathrm{GPa})\end{array}$ & $V / V_{0}$ & $\begin{array}{c}\rho \\
\left(\mathrm{g} / \mathrm{cm}^{3}\right)\end{array}$ & std & $\begin{array}{c}u_{\text {sstd }} \\
(\mathrm{km} / \mathrm{s})\end{array}$ \\
\hline 1.205 & 2.270 & 0.000 & 0.0 & 1.000 & 1.205 & 40 & $\overline{0.00}$ \\
\hline 1.207 & 3.404 & 0.710 & 2.9 & 0.791 & 1.525 & 1 & 5.93 \\
\hline 1.199 & 3.720 & 0.735 & 3.3 & 0.902 & 1.494 & 1 & 5.96 \\
\hline 1.209 & 3.583 & 0.799 & 3.5 & 0.777 & 1.556 & 1 & 6.01 \\
\hline 1.208 & 3.738 & 0.906 & 4.1 & 0.758 & 1.594 & 1 & 6.11 \\
\hline 1.209 & 3.945 & 0.960 & 4.6 & 0.757 & 1.598 & 1 & 6.16 \\
\hline 1.206 & 4.530 & 1.365 & 7.5 & 0.699 & 1.726 & 1 & 6.54 \\
\hline 1.205 & 5.025 & 1.767 & 10.7 & 0.648 & 1.859 & 1 & 6.92 \\
\hline 1.206 & 5.129 & 1.776 & 11.0 & 0.654 & 1.845 & 1 & 6.94 \\
\hline 1.210 & 6.059 & 2.427 & 17.8 & 0.599 & 2.019 & 1 & 7.59 \\
\hline 1.205 & 5.981 & 2.447 & 17.6 & 0.591 & 2.039 & 1 & 7.60 \\
\hline 1.208 & 5.808 & 2.453 & 17.2 & 0.578 & 2.091 & 1 & 7.59 \\
\hline 1.206 & 5.821 & 2.463 & 17.3 & 0.577 & 2.090 & 1 & 7.60 \\
\hline 1.198 & 5.918 & 2.610 & 18.5 & 0.559 & 2.144 & 1 & 7.73 \\
\hline 1.204 & 5.962 & 2.618 & 18.8 & 0.561 & 2.146 & 1 & 7.75 \\
\hline 1.206 & 6.055 & 2.862 & 20.9 & 0.527 & 2.287 & 1 & 7.97 \\
\hline 1.204 & 6.174 & 3.095 & 23.0 & 0.499 & 2.415 & 1 & 8.19 \\
\hline 1.209 & 6.352 & 3.135 & 24.1 & 0.506 & 2.387 & 1 & 8.24 \\
\hline 1.209 & 6.515 & 3.359 & 26.5 & 0.484 & 2.496 & 1 & 8.46 \\
\hline 1.209 & 6.839 & 3.629 & 30.0 & 0.469 & 2.576 & 1 & 8.73 \\
\hline 1.209 & 7.505 & 4.088 & 37.1 & 0.455 & 2.655 & 1 & 9.21 \\
\hline 1.209 & 8.000 & 4.354 & 42.1 & 0.456 & 2.653 & 1 & 9.51 \\
\hline 1.209 & 8.357 & 4.622 & 46.7 & 0.447 & 2.705 & 1 & 9.79 \\
\hline 1.209 & 8.666 & 4.837 & 50.7 & 0.442 & 2.736 & 1 & 10.02 \\
\hline 1.209 & 9.274 & 5.182 & 58.1 & 0.441 & 2.740 & 1 & 10.40 \\
\hline 1.209 & 9.178 & 5.225 & 58.0 & 0.431 & 2.807 & 1 & 10.43 \\
\hline 1.209 & 9.178 & 5.225 & 58.0 & 0.431 & 2.807 & 1 & 10.43 \\
\hline
\end{tabular}


Polysulfone (P 1700-Union Carbide)

\begin{tabular}{rccccrrr}
\hline $\begin{array}{c}\rho_{o} \\
\left(\mathrm{~g} / \mathrm{cm}^{3}\right)\end{array}$ & $\begin{array}{c}u_{s} \\
(\mathrm{~km} / \mathrm{s})\end{array}$ & $\begin{array}{c}u_{p} \\
(\mathrm{~km} / \mathrm{s})\end{array}$ & $\begin{array}{c}P \\
(\mathrm{GPa})\end{array}$ & & $\begin{array}{c}\rho \\
\text { ( })\end{array}$ & $\begin{array}{r}\text { std } \\
\left(\mathrm{g} / \mathrm{cm}^{3}\right)\end{array}$ & $\begin{array}{c}u_{\text {sstd }} \\
(\mathrm{km} / \mathrm{s})\end{array}$ \\
\hline 1.235 & 1.980 & 0.000 & 0.0 & 1.000 & 1.235 & 40 & 0.00 \\
1.235 & 3.482 & 0.729 & 3.1 & 0.791 & 1.562 & 1 & 5.95 \\
1.235 & 3.434 & 0.729 & 3.1 & 0.788 & 1.568 & 1 & 5.95 \\
1.235 & 3.869 & 0.957 & 4.6 & 0.753 & 1.641 & 1 & 6.16 \\
1.235 & 3.868 & 1.001 & 4.8 & 0.741 & 1.666 & 1 & 6.20 \\
1.235 & 4.576 & 1.384 & 7.8 & 0.698 & 1.771 & 1 & 6.57 \\
1.235 & 4.494 & 1.385 & 7.7 & 0.692 & 1.785 & 1 & 6.56 \\
1.235 & 5.125 & 1.766 & 11.2 & 0.655 & 1.884 & 1 & 6.94 \\
1.235 & 5.119 & 1.799 & 11.4 & 0.649 & 1.904 & 1 & 6.97 \\
1.235 & 6.113 & 2.418 & 18.3 & 0.604 & 2.043 & 1 & 7.60 \\
1.235 & 6.111 & 2.419 & 18.3 & 0.604 & 2.046 & 1 & 7.60 \\
1.235 & 6.104 & 2.444 & 18.4 & 0.600 & 2.060 & 1 & 7.62 \\
1.235 & 6.287 & 2.591 & 20.1 & 0.598 & 2.101 & 1 & 7.77 \\
1.236 & 6.311 & 2.617 & 20.4 & 0.585 & 2.112 & 1 & 7.79 \\
1.236 & 6.407 & 2.827 & 22.4 & 0.559 & 2.212 & 1 & 7.99 \\
1.236 & 6.549 & 3.153 & 25.5 & 0.519 & 2.383 & 1 & 8.29 \\
1.235 & 6.580 & 3.212 & 26.1 & 0.512 & 2.413 & 1 & 8.35 \\
1.235 & 6.729 & 3.397 & 28.2 & 0.495 & 2.494 & 1 & 8.53 \\
1.235 & 6.995 & 3.559 & 30.7 & 0.491 & 2.515 & 1 & 8.70 \\
1.235 & 7.034 & 3.652 & 31.7 & 0.481 & 2.568 & 1 & 8.79 \\
1.235 & 7.421 & 3.840 & 35.2 & 0.483 & 2.559 & 1 & 9.00 \\
1.235 & 7.983 & 4.224 & 41.6 & 0.471 & 2.623 & 1 & 9.41 \\
1.235 & 8.493 & 4.573 & 48.0 & 0.462 & 2.675 & 1 & 9.78 \\
1.235 & 8.751 & 4.752 & 51.4 & 0.457 & 2.703 & 1 & 9.98 \\
1.235 & 9.295 & 5.100 & 58.5 & 0.451 & 2.736 & 1 & 10.36 \\
\hline \hline
\end{tabular}

Polyurethane (CPR Polycast 1009-78)

\begin{tabular}{rccccrrr}
\hline $\begin{array}{c}\rho_{o} \\
\left(\mathrm{~g} / \mathrm{cm}^{3}\right)\end{array}$ & $\begin{array}{c}u_{s} \\
(\mathrm{~km} / \mathrm{s})\end{array}$ & $\begin{array}{c}u_{p} \\
(\mathrm{~km} / \mathrm{s})\end{array}$ & $\begin{array}{c}P \\
(\mathrm{GPa})\end{array}$ & $V / V_{o}$ & $\begin{array}{c}\rho \\
\left(\mathrm{g} / \mathrm{cm}^{3}\right)\end{array}$ & std & $\begin{array}{r}u_{\text {sstd }} \\
(\mathrm{km} / \mathrm{s})\end{array}$ \\
\hline 1.265 & 2.068 & 0.000 & 0.0 & 1.000 & 1.265 & 40 & 0.00 \\
1.262 & 3.644 & 0.659 & 3.0 & 0.819 & 1.541 & 1 & 5.90 \\
1.262 & 4.007 & 0.983 & 5.0 & 0.755 & 1.672 & 1 & 6.19 \\
1.262 & 4.681 & 1.358 & 8.0 & 0.710 & 1.778 & 1 & 6.56 \\
1.262 & 5.269 & 1.762 & 11.7 & 0.666 & 1.896 & 1 & 6.96 \\
1.262 & 6.298 & 2.376 & 18.9 & 0.623 & 2.027 & 1 & 7.59 \\
1.262 & 6.570 & 2.565 & 21.3 & 0.610 & 2.071 & 1 & 7.79 \\
1.262 & 6.795 & 2.821 & 24.2 & 0.585 & 2.158 & 1 & 6.04 \\
1.262 & 7.099 & 3.100 & 27.8 & 0.563 & 2.240 & 1 & 8.32 \\
1.262 & 7.177 & 3.273 & 29.6 & 0.544 & 2.320 & 1 & 8.49 \\
1.262 & 7.389 & 3.505 & 32.7 & 0.526 & 2.401 & 1 & 8.72 \\
1.265 & 7.836 & 3.749 & 37.2 & 0.522 & 2.425 & 1 & 8.99 \\
1.262 & 7.801 & 3.844 & 37.8 & 0.507 & 2.488 & 1 & 9.07 \\
1.262 & 8.975 & 4.542 & 51.4 & 0.494 & 2.555 & 1 & 9.84 \\
1.265 & 8.877 & 4.602 & 51.7 & 0.482 & 2.627 & 1 & 9.89 \\
1.262 & 2.019 & 4.610 & 52.5 & 0.489 & 2.581 & 1 & 9.91 \\
1.262 & 9.245 & 4.750 & 55.4 & 0.486 & 2.596 & 1 & 10.06 \\
1.265 & 9.516 & 5.009 & 60.3 & 0.474 & 2.671 & 1 & 10.33 \\
1.262 & 9.764 & 5.066 & 62.4 & 0.481 & 2.623 & 1 & 10.41 \\
\hline \hline
\end{tabular}

